Astrophysical Journal, in press

Preprint typeset using $\mathrm{AT}_{\mathrm{E} X} \mathrm{X}$ style emulateapj v. 11/10/09

\title{
A CENSUS OF THE HIGH-DENSITY MOLECULAR GAS IN M82
}

\author{
B.J. NAYlor ${ }^{1}$, C.M. Bradford ${ }^{1}$, J.E. Aguirre ${ }^{2}$, J.J. Bock ${ }^{1}$, L. Earle ${ }^{3}$, J. Glenn ${ }^{3}$, H. InAmi ${ }^{4}$, J. KAmenetzkY $^{3}$, P.R. \\ Maloney $^{3}$, H. Matsuhara ${ }^{4}$, H.T. NGUYen ${ }^{1}$, J. ZMuidzinas ${ }^{1}$ \\ Astrophysical Journal, in press
}

\begin{abstract}
We present a three-pointing study of the molecular gas in the starburst nucleus of M82 based on 190$307 \mathrm{GHz}$ spectra obtained with Z-Spec at the Caltech Submillimeter Observatory. We present intensity measurements, detections and upper limits, for 20 transitions, including several new detections of CS, $\mathrm{HNC}, \mathrm{C}_{2} \mathrm{H}, \mathrm{H}_{2} \mathrm{CO}$, and $\mathrm{CH}_{3} \mathrm{CCH}$ lines. We combine our measurements with previously-published measurements at other frequencies for $\mathrm{HCN}, \mathrm{HNC}, \mathrm{CS}, \mathrm{C}^{34} \mathrm{~S}$, and $\mathrm{HCO}^{+}$in a multi-species likelihood analysis constraining gas mass, density and temperature, and the species' relative abundances. We find some $1.7-2.7 \times 10^{8} M_{\odot}$ of gas with $n_{\mathrm{H}_{2}}$ between $1-6 \times 10^{4} \mathrm{~cm}^{-3}$ and $T>50 \mathrm{~K}$. While the mass and temperature are comparable to values inferred from mid- $J$ CO transitions, the thermal pressure is a factor of 10-20 greater. The molecular interstellar medium is largely fragmented and is subject to ultraviolet irradiation from the star clusters. It is also likely subject to cosmic rays and mechanical energy input from the supernovae, and is warmer on average than the molecular gas in the massive star formation regions in the Milky Way. The typical conditions in the dense gas in M82's central $\mathrm{kpc}$ appear unfavorable for further star formation; if any appreciable stellar populations are currently forming, they are likely biased against low mass stars, producing a top-heavy initial mass function.

Subject headings: galaxies: abundances — galaxies: individual (M82) — galaxies: ISM — galaxies: starburst - instrumentation: spectrographs - techniques: spectroscopic
\end{abstract}

\section{INTRODUCTION}

Studies of molecular gas - the actively star-forming part of the interstellar medium (ISM) - in other galaxies have been carried out most extensively in the low-lying rotational transitions of the $\mathrm{CO}$ molecule. Due to its high abundance relative to molecular hydrogen and its favorable energy level spacing as compared to typical molecular cloud temperatures, $\mathrm{CO}$ produces the brightest lines in the millimeter-wavelength portion of the spectrum.

However, while these lines trace the bulk of the molecular gas, the small dipole moment of $\mathrm{CO}$ results in modest critical densities for these lines, $10^{3}-10^{4} \mathrm{~cm}^{-3}$, and thus they do not strongly discriminate between high-density star-forming cores and more diffuse gas. High-dipole-moment molecules, such as $\mathrm{HCN}$ and $\mathrm{CS}$, have critical densities and radiative rates that are $100-1000$ times larger than CO. Despite their much smaller abundances, these species can be used to probe the dense $\left(10^{4}-10^{7} \mathrm{~cm}^{-3}\right)$ cloud cores believed to be associated with star formation (SF). Early measurement of HCN and CS in the Galaxy (Morris et al. 1974; Turner et al. 1973) and in external galaxies (Henkel et al. 1988; Solomon et al. 1992) showed that their intensities are well-correlated with the total far-infrared (FIR) flux. More recently, $\mathrm{HCN} J=1 \rightarrow 0$ luminosity has been shown to be directly proportional to FIR luminosity (a proxy for star formation rate [SFR]) in a sample of $~ 100$ normal spiral and starburst galaxies (Gao \& Solomon 2004a b), as well as individual star-

\footnotetext{
${ }^{1}$ Jet Propulsion Laboratory, California Institute of Technology, Pasadena, CA, 91109

2 University of Pennsylvania, Philadelphia, PA 19104

3 Dept. of Astrophysical and Planetary Sciences, University of Colorado, 389-UCB, Boulder, CO 80309

${ }^{4}$ ISAS / JAXA, Sagamihara, Japan
}

formation sites in the Galaxy (Wu et al. 2005).

Insofar as HCN $J=1 \rightarrow 0$ measures dense gas mass, the $L_{\text {FIR }} / \mathrm{HCN}$ correlation across $7-8$ orders of magnitude in luminosity implies a scale-independent relationship between dense gas mass and SFR. An accurate assessment of the physical conditions in the HCN-emitting gas as well as the mass scaling $\left(M_{\mathrm{H}_{2}} / I_{\mathrm{HCN}}\right)$ is thus of universal interest for theoretical SF studies. Is the HCNemitting gas simply a bi-product of star formation, perhaps formed in shocks or outflows and not participating in the formation of new stars, or is some of it the very material from which new stars form? Such an assessment is best made with multiple transitions of $\mathrm{HCN}$ and by including transitions from other high-dipole-moment molecules where available.

To assess the average properties of the dense gas on the scale of a nuclear starburst, we have observed the nucleus of the M82 in a suite of millimeter-wave transitions of high-dipole moment species. The brightest infrared (IR) galaxy in the sky due to its proximity $(3.9 \mathrm{Mpc}$, Sakai \& Madore 1999), M82 radiates an infrared luminosity $\left(L=5.9 \times 10^{10} L_{\odot}\right.$, Sanders et al. 2003), exceeding that of the Galaxy, from a region that is only about 450 pc in radius (e.g., Leeuw \& Robson 2009). Because of this concentration of star-forming activity, M82 has been dubbed the prototypical starburst galaxy, which makes it a particularly interesting laboratory for the study of SF. It has been suggested for over 30 years that the stellar initial mass function (IMF) in M82 (and presumably other starburst nuclei) is biased against low-mass stars relative to the the local IMF (Rieke et al. 1980, 1993; Förster Schreiber et al. 2003), but this has been debated (e.g., Satyapal et al. 1997; Colbert et al. 1999). If the IMF is indeed low-mass deficient, a plausible line of inquiry is the initial conditions of $\mathrm{SF}$ - the temperature 
and density of the dense molecular cloud cores.

We have obtained full $190-307 \mathrm{GHz}$ spectra with the Z-Spec 1-mm grating spectrometer, which accesses the $J=3 \rightarrow 2$ transitions of $\mathrm{HCN}, \mathrm{HCO}^{+}$, and $\mathrm{HNC}$ and $J=4 \rightarrow 3, J=5 \rightarrow 4$, and $J=6 \rightarrow 5$ transitions of CS, with a uniform calibration. Our study benefits from the prior observations of $J=1 \rightarrow 0$ transitions of $\mathrm{HCN}, \mathrm{HNC}, \mathrm{HCO}^{+}$(Nguyen-Q-Rieu et al. 1989; Huettemeister et al. 1995), as well as $J=4 \rightarrow 3$ transitions of $\mathrm{HCN}, \mathrm{HCO}^{+}$(Seaquist \& Frayer 2000). These data are combined with our observations to generate the first comprehensive multi-species excitation and radiative transfer model for the dense gas in this source. Of course, since M82 is also well-studied in multiple CO transitions (Wild et al. 1992; Mao et al. 2000; Ward et al. 2003; Weiß et al. 2005; Seaquist et al. 2006; Panuzzo et al. 2010) as well as in the mid- and far-IR atomic gas tracers (Förster Schreiber et al. 2003; Colbert et al. 1999), we have the opportunity to put the dense gas into context with the other ISM components, as well as the general properties of this prototypical nuclear starburst.

\section{OBSERVATIONS}

Z-Spec is the first grating spectrometer for the millimeter band; it covers the full $190-307 \mathrm{GHz}$ range instantaneously, dispersing this band to an array of 160 bolometers. More information can be found in Glenn et al. (2007), Bradford et al. (2009) and the Society of Photo-Optical Instrumentation Engineers (SPIE) articles: (Navlor et al. 2003; Bradford et al. 2004; Earle et al. 2006). The instrument operates at the Nasmyth focus of the Caltech Submillimeter Observatory (CSO) atop Mauna Kea. While the instrument is $1 / f$ stable down to $\sim 100 \mathrm{mHz}$, we use a chop and nod mode to avoid the atmospheric fluctuations, which become important relative to the fundamental noise sources at $\sim 0.3-1 \mathrm{~Hz}$, depending on the atmospheric conditions. For the M82 observations the chop frequency was $1.6 \mathrm{~Hz}$, the throw was $90^{\prime \prime}$ in azimuth, and the nod interval was 20 seconds. Three pointings along M82's major axis were observed on 2009 January 5 as summarized in Figure 1 and Table 1. The M82 spectra are calibrated using Mars, with an interpolation scheme using bolometer operating voltages as a measure of response Earle 2008; Bradford et al. 2009). The data were reduced with standard demodulation and differencing appropriate for the chop and nod observing mode and Uranus is used as a spectral flat-fielder. We expect the channel-to-channel calibration uncertainties to be less that $10 \%$ except at the lowest frequencies which are extremely sensitive to the wing of the $186 \mathrm{GHz}$ atmospheric water line.

\section{RESULTS}

Spectra for the NE, CEN, and SW pointings are shown in Figure 2. The general features agree with previous measurements of the $1.2-\mathrm{mm}$ continuum and $\mathrm{CO} J=$ $2 \rightarrow 1$, such as those in Figure 1.

\subsection{Continuum Fluxes and Fits}

The continuum flux in the Z-Spec band is due to a combination of the thermal dust emission which dominates at higher frequencies and the free-free emission from $[\mathrm{H}$
Table 1

M82 Observed Positions

\begin{tabular}{ccccc}
\hline \hline Pointing & $\begin{array}{c}\alpha \text { offset } \\
(\operatorname{arcsec})\end{array}$ & $\begin{array}{c}\delta \text { offset } \\
(\operatorname{arcsec})\end{array}$ & $\begin{array}{c}\text { Int. Time } \\
(\min )\end{array}$ & $\begin{array}{c}\text { Sensitivity } \\
\left(\mathrm{Jy} \mathrm{s}^{1 / 2}\right)\end{array}$ \\
\hline $\mathrm{NE}$ & +12.2 & +3.3 & 60.2 & 1.3 \\
$\mathrm{CEN}$ & +2.7 & -0.5 & 68.6 & 1.4 \\
$\mathrm{SW}$ & -6.1 & -3.8 & 60.1 & 1.2 \\
\hline
\end{tabular}

Note. - The R. A. and Dec. offsets are relative to $\alpha_{J 2000.0}=9^{\mathrm{h}} 55^{\mathrm{m}} 51.9^{\mathrm{s}}, \delta_{J 2000.0}=69^{\circ} 40^{\prime} 47.14^{\prime \prime}$. The integration time is the total demodulated time; the onsource time is half the listed values. The quoted sensitivity is the median value of channel errors multiplied by the square root of the integration time and does not represent the ultimate sensitivity of the instrument; Z-Spec's sensitivity to spectral lines and to fainter sources is better by at least a factor of two. The optical depth during these observations was $\tau_{225 \mathrm{GHz}}=0.08-0.1$.

II] region electrons which dominates at lower frequencies. The dust emission is parameterized as

$$
F_{\mathrm{T}}(\nu)=\Omega B_{\nu}(T)\left\{1-\exp \left[-\left(\frac{\lambda_{0}}{\lambda}\right)^{\beta}\right]\right\},
$$

where $B_{\nu}(T)=\left(2 h \nu^{3} / c^{2}\right) /(\exp [h \nu / k T]-1)$ is the Planck function. Hughes et al. (1994) fit a collection of observations from $3.3 \mathrm{~mm}$ to $40 \mu \mathrm{m}$ and derive $\Omega=1.34 \times 10^{-8}$ sr, $T=48.1 \mathrm{~K}, \beta=1.3$ and $\lambda_{0}=7.9$ microns for the thermal dust. The free-free emission is given by a simple power law,

$$
F_{\mathrm{ff}}(\nu)=F_{0}\left(\frac{\nu}{\nu_{0}}\right)^{-0.1},
$$

where $F_{0}$ is the free-free flux at $\nu_{0}$. Analysis of a map of M82 at $92 \mathrm{GHz}$ found that the emission at that frequency is dominated by free-free emission. Carlstrom \& Kronberg (1991) calculate the free-free flux density $F_{\mathrm{ff}}(92 \mathrm{GHz})=0.5 \pm 0.1 \mathrm{Jy}$. In the Z-Spec band, the free-free emission accounts for roughly one quarter of the total continuum flux, but that is significant enough that a dust-only fit to our spectra does not match the continuum level. Instead, we model the continuum flux we observe to be a beam-scaled fraction of the total continuum emission of the galaxy,

$$
F(\nu)=A\left(\frac{\nu}{240 \mathrm{GHz}}\right)^{B-2}\left[F_{\mathrm{T}}(\nu)+F_{\mathrm{ff}}(\nu)\right] .
$$

This model assumes that the free-free and thermal continuum emission have the same spatial distribution, which is reasonable given our coarse spatial resolution. It also accounts for the spectral dependence caused by the spatial distribution. If M82 completely filled our beam at all frequencies, we would expect $B=0$, while $B=2$ would be the prediction for a point source. We find that $B=1$ is the best fit value for all three pointings (see Table 2), which makes sense given that M82 is observed edge on and is thus roughly point-like in one dimension and beam-filling in the other, relative to our $30^{\prime \prime}$ beam. The continuum fraction seen in the three pointings is consistent with the $1.2 \mathrm{~mm}$ continuum distribution shown in Thuma et al. (2000). The peak of the $1.2 \mathrm{~mm}$ continuum is at the center of the SW pointing which has the largest continuum fraction and smallest beam-scaling exponent 


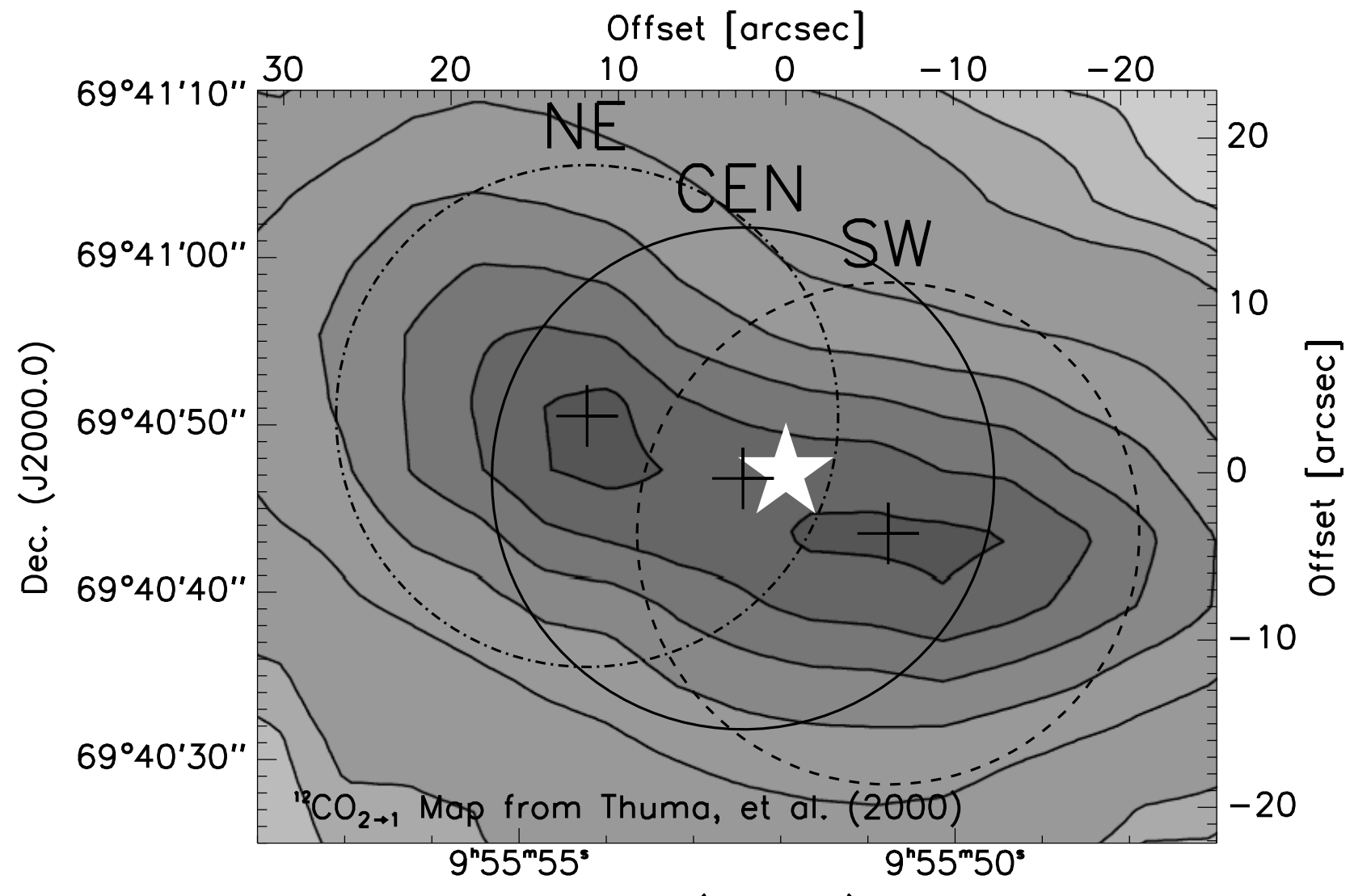

R.A. (J2000.0)

Figure 1. Pointings toward M82. Z-Spec's $30^{\prime \prime}$ beam (FWHM at $250 \mathrm{GHz}$ ) is plotted over the single-dish measurements of the ${ }^{12} \mathrm{CO}$ $J=2 \rightarrow 1$ map from Thuma et al. (2000). The white star marks the $2.2 \mu \mathrm{m}$ peak at $\alpha_{J 2000.0}=9^{\mathrm{h}} 55^{\mathrm{m}} 51.9^{\mathrm{s}}, \delta_{J 2000.0}=69^{\circ} 40^{\prime} 47.14^{\prime \prime}$ which is the adopted center for the observations. The offsets relative to the center and integration times are given in Table 1 All three pointings use a $90^{\prime \prime}$ symmetric azimuthal chop throw.

Table 2

Continuum Fit Results

\begin{tabular}{cccc}
\hline \hline Pointing & \multicolumn{2}{c}{ Continuum Fit } & Reduced $\chi^{2}$ \\
& $A$ & $B$ & \\
\hline NE & $0.318 \pm 0.001$ & $1.03 \pm 0.03$ & 2.4 \\
CEN & $0.413 \pm 0.001$ & $1.05 \pm 0.02$ & 3.2 \\
SW & $0.425 \pm 0.001$ & $0.99 \pm 0.02$ & 3.7 \\
\hline
\end{tabular}

Note. - The continuum fit columns give the coupling fraction $(A)$ and beam scaling exponent $(B)$ of the fit described in Section 3.1 and defined in equation 3 The reduced $\chi^{2}$ values are for the line and continuum fits and are calculated based solely on statistical errors and do not include errors due to calibration. The fits use all data points except the lowest five channels in each spectrum and have 132 degrees of freedom.

of the three pointings. The continuum peak is within the CEN pointing and its fraction is almost equal to the SW but with a higher index. The NE pointing's continuum fraction is the smallest because it is off the peak.

\subsection{Spectral Line Fitting Results}

The channel-to-channel spacing in Z-Spec is 500$1300 \mathrm{MHz}$, corresponding to $700-1200 \mathrm{~km} \mathrm{~s}^{-1}$, thus the instrument does not resolve the line profiles in M82. Nev- ertheless, it is possible to fit integrated intensities and center frequencies by comparison with the instrumental response for each bolometer, carefully measured in the laboratory with a long-path Fourier-transform spectrometer. Each spectral line (indexed by $j$ ) is modeled as a Gaussian profile of center frequency $\nu_{j}$, amplitude $A_{j}$, and FHWM $\delta \nu_{j}$. The sum of these line profiles plus the continuum is multiplied by the (normalized) measured spectral profile of each channel used in the fitting (indexed by $i) f_{i}(\nu)$ and integrated over the range of the measured profiles: $180-320 \mathrm{GHz}$. This process creates a model Z-Spec spectrum, which can be compared to the observed spectrum. The input line frequencies and amplitudes are then varied to arrive at a least-squares fit, using statistical $\left(1 / \sigma_{i}^{2}\right)$ weighting. The frequencies of the known lines are fixed in the fitting, modulo a common redshift, so that the fit determines the redshift but not the line frequencies. In practice for local-Universe galaxies, the ${ }^{12} \mathrm{CO}$ transition dominates the redshift determination. With this method, we obtain accurate centroid measurements of line features that did not have an obvious identification. The fitted line intensities are given in Table 3 using the adopted $\Delta v=250 \mathrm{~km} \mathrm{~s}^{-1}$ for all lines in the three pointings; reducing the FWHM to 100 $\mathrm{km} \mathrm{s}^{-1}$ produces nearly identical integrated intensities 


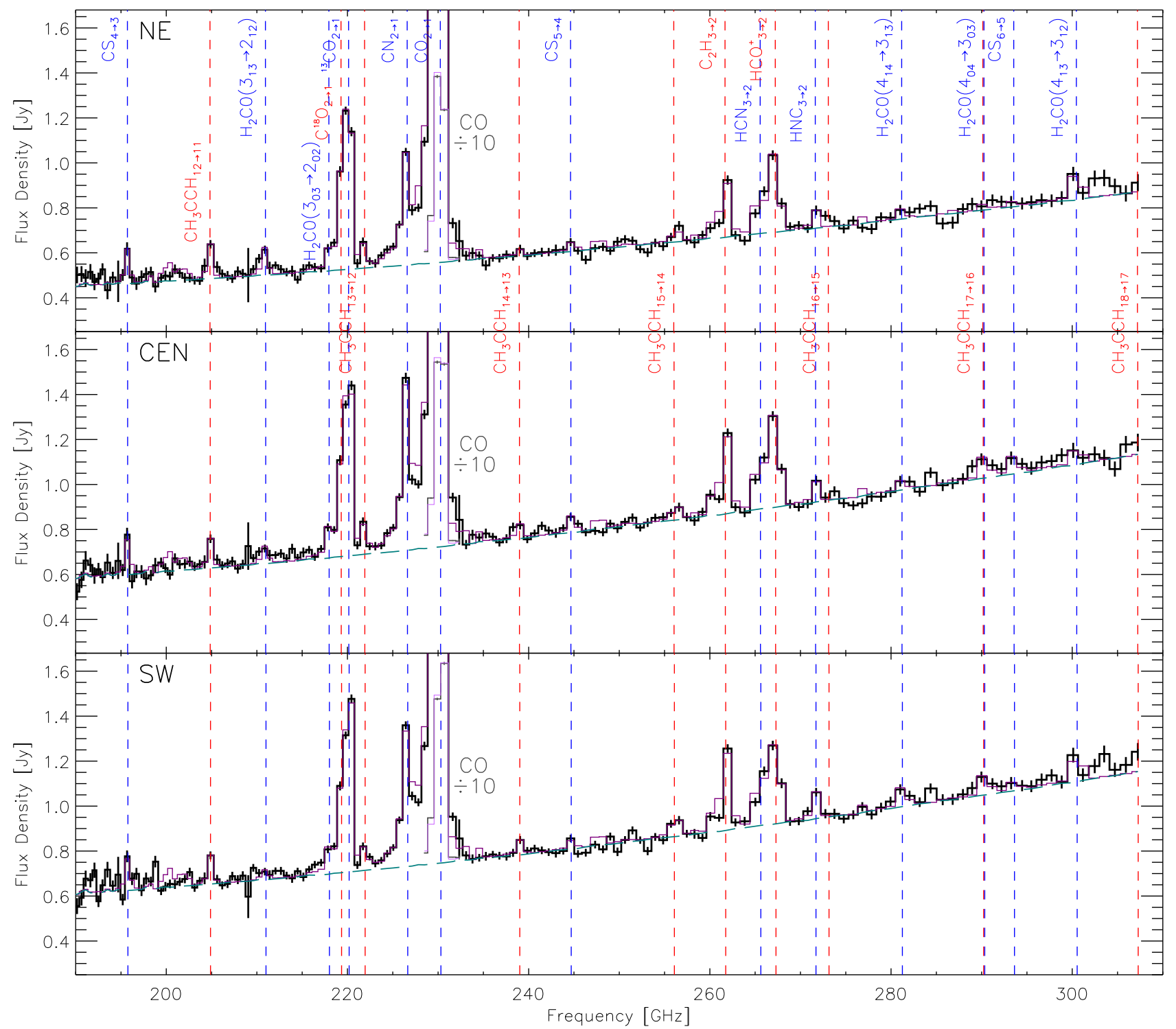

Figure 2. Z-Spec 190-307 GHz spectra toward three positions in the nucleus of M82: NE at the top, CEN in the middle, and SW on the bottom. The black histogram with error bars are the calibrated measurements and the purple histogram is the spectral fit, including both continuum and 20 fitted lines. The fitted continuum is also plotted in dashed dark green and the fitted lines are marked with vertical blue and red dashed lines. The dominant $\mathrm{CO} J=2 \rightarrow 1$ line is shown in gray on the plot, scaled down by a factor of ten; the fit to the CO line, also scaled down, is shown in lavender. The $\mathrm{CH}_{3} \mathrm{CCH} J=16 \rightarrow 15$ transitions and above are shown for reference but are not included in the fit. We have identified all three sigma or greater spectral-line features that appear in all three pointings. Nonetheless, there is additional structure in the spectra probably due to a multitude of blended, weak lines which Z-spec cannot individually identify. The results from the fits are given in in Tables 2 and 3

and fit quality. We comment here on our spectral line measurements and how they compare to those found in the literature.

\subsection{1. $C O$ and its Isotopologues}

The $\mathrm{CO},{ }^{13} \mathrm{CO}$, and $\mathrm{C}^{18} \mathrm{O} J=2 \rightarrow 1$ transitions have been extensively studied and mapped by previous experiments (Mao et al. 2000; Weiß et al. 2001); however, direct comparison with published intensities is limited by beam size mismatch. Wild et al. (1992) quote inte- grated main-beam intensities nearly twice what we measure in a $13^{\prime \prime}$ beam for the CO $J=2 \rightarrow 1$ transition, implying a beamsize $(\theta)$ scaling of $I \propto \theta^{-0.8}$ whereas the main species intensities given in Mao et al. (2000) in a $22^{\prime \prime}$ beam indicate a beam scaling exponent of -1.2 to -1.5 . The maps obtained by Thuma et al. (2000) show that the $\mathrm{CO}$ emission is more extended than the continuum emission which would indicate the exponent should be slightly less than unity. The isotopologues ${ }^{13} \mathrm{CO}$ and $\mathrm{C}^{18} \mathrm{O}$ have been measured in Mao et al. (2000) 
High-Density Molecular Gas in M82

Table 3

M82 Line Fit Results

\begin{tabular}{|c|c|c|c|c|c|c|}
\hline $\begin{array}{l}\text { Species \& } \\
\text { Transition }\end{array}$ & $\begin{array}{l}\text { Rest Freq. } \\
(\mathrm{GHz})\end{array}$ & $\begin{array}{l}E_{\text {upper }} \\
(\mathrm{K})\end{array}$ & $\begin{array}{l}\text { Beam Size } \\
(\operatorname{arcsec})\end{array}$ & $\begin{array}{l}\text { Integrated } \\
\text { NE }\end{array}$ & $\begin{array}{c}\text { Line Intensity } \\
\text { CEN }\end{array}$ & $\left(\begin{array}{c}\left.\mathrm{K} \mathrm{km} \mathrm{s}^{-1}\right) \\
\mathrm{SW}\end{array}\right.$ \\
\hline $\mathrm{CO} J=2 \rightarrow 1$ & 230.538 & 16.6 & 32 & $423.9 \pm 3.0$ & $460.0 \pm 3.2$ & $452.5 \pm 3.3$ \\
\hline${ }^{13} \mathrm{CO} J=2 \rightarrow 1$ & 220.399 & 15.9 & 34 & $29.4 \pm 0.7$ & $31.7 \pm 0.7$ & $30.2 \pm 0.7$ \\
\hline $\mathrm{C}^{18} \mathrm{O} J=2 \rightarrow 1$ & 219.560 & 15.8 & 34 & $8.6 \pm 0.7$ & $7.9 \pm 0.7$ & $7.8 \pm 0.7$ \\
\hline $\mathrm{CN} J=2 \rightarrow 1$ & 226.875 & 16.3 & 33 & $14.3 \pm 0.6$ & $23.3 \pm 0.8$ & $19.9 \pm 0.6$ \\
\hline CS $J=4 \rightarrow 3$ & 195.954 & 23.5 & 37 & $4.3 \pm 0.8$ & $4.4 \pm 0.8$ & $4.1 \pm 0.7$ \\
\hline $\mathrm{CS} J=5 \rightarrow 4$ & 244.936 & 35.3 & 31 & $<1.6[1.2]$ & $2.3 \pm 0.5$ & $<1.5[1.1]$ \\
\hline $\mathrm{CS} J=6 \rightarrow 5$ & 293.912 & 49.4 & 26 & $<3.1[0.8]$ & $<3.3[3.0]$ & $<3.1[1.5]$ \\
\hline $\mathrm{HCO}^{+} J=3 \rightarrow 2$ & 267.558 & 25.7 & 29 & $13.7 \pm 0.8$ & $16.9 \pm 0.9$ & $15.4 \pm 0.9$ \\
\hline $\mathrm{HCN} J=3 \rightarrow 2$ & 265.886 & 25.5 & 29 & $5.1 \pm 0.8$ & $7.0 \pm 0.8$ & $6.9 \pm 0.8$ \\
\hline HNC $J=3 \rightarrow 2$ & 271.981 & 26.1 & 28 & $2.4 \pm 0.6$ & $2.7 \pm 0.7$ & $3.5 \pm 0.6$ \\
\hline $\mathrm{C}_{2} \mathrm{H} \quad J=3 \rightarrow 2$ & 262.251 & 25.2 & 29 & $7.9 \pm 0.7$ & $11.1 \pm 0.7$ & $11.0 \pm 0.6$ \\
\hline $\mathrm{H}_{2} \mathrm{CO}\left(3_{13} \rightarrow 2_{12}\right)$ & 211.211 & 32.1 & 35 & $4.0 \pm 0.6$ & $2.6 \pm 0.6$ & $<1.6[1.5]$ \\
\hline $\mathrm{H}_{2} \mathrm{CO}\left(3_{03} \rightarrow 202\right)^{*}$ & 218.222 & 21.0 & 34 & $2.3 \pm 0.6$ & $3.6 \pm 0.6$ & $3.1 \pm 0.6$ \\
\hline $\mathrm{H}_{2} \mathrm{CO}\left(4_{14} \rightarrow 3_{13}\right)$ & 281.527 & 45.6 & 27 & $<2.5[1.7]$ & $<2 . \overline{6}[1.6]$ & $3.3 \pm 0.9$ \\
\hline $\mathrm{H}_{2} \mathrm{CO}\left(4_{04} \rightarrow 3_{03}\right)^{*}$ & 290.623 & 34.9 & 27 & $<3.1[1.0]$ & $3.7 \pm 1.0$ & $3.6 \pm 1.0$ \\
\hline $\mathrm{H}_{2} \mathrm{CO}\left(4_{13} \rightarrow 3_{12}\right)$ & 300.837 & 47.9 & 26 & $4.0 \pm 1.1$ & $<3.5[2.6]$ & $4.0 \pm 1.1$ \\
\hline $\mathrm{CH}_{3} \mathrm{CCH} J=12 \rightarrow 11$ & 205.081 & 64.0 & 36 & $5.1 \pm 0.6$ & $4.3 \pm 0.6$ & $4.2 \pm 0.6$ \\
\hline $\mathrm{CH}_{3} \mathrm{CCH} J=13 \rightarrow 12$ & 222.167 & 74.6 & 33 & $2.5 \pm 0.7$ & $3.4 \pm 0.7$ & $2.5 \pm 0.7$ \\
\hline $\mathrm{CH}_{3} \mathrm{CCH} J=14 \rightarrow 13$ & 239.252 & 86.1 & 31 & $<1.9[1.0]$ & $2.2 \pm 0.6$ & $2.1 \pm 0.6$ \\
\hline $\mathrm{CH}_{3} \mathrm{CCH} J=15 \rightarrow 14$ & 256.337 & 98.4 & 30 & $<2.2[1.0]$ & $<2.2[1.4]$ & $2.3 \pm 0.7$ \\
\hline
\end{tabular}

Note. - Measured line intensities and their estimated uncertainties or the three- $\sigma$ upper limits for the 20 identified transitions in the three pointings. For for the upper limits, the value is three times the $1-\sigma$ uncertainty in the fitted intensity and the fitted intensity value is given in square brackets. The adopted linewidth for all measurements is $250 \mathrm{~km} \mathrm{~s}^{-1}$ (see text); changing this value by factors of two in either direction does not significantly change the fitted line intensities or the quality of the fit. The errors are based on the statistical errors in the spectral data and do not include any uncertainty due to calibration. The hyperfine splitting in the $\mathrm{CN}$ and $\mathrm{C}_{2} \mathrm{H}$ transitions is not accessible at Z-Spec's resolution. The $\mathrm{H}_{2} \mathrm{CO}$ lines marked with an * may be blended with other higher energy $\mathrm{H}_{2} \mathrm{CO}$ transitions.

and Wild et al. (1992), respectively, and though they indicate different beam scaling exponents as they did for the main species, they both imply that the isotopologue distribution is slightly more concentrated than the main species.

\subsection{2. $\mathrm{CN}$ and $\mathrm{C}_{2} \mathrm{H}$}

We detect both the $\mathrm{CN} J=2 \rightarrow 1$ and $\mathrm{C}_{2} \mathrm{H} J=3 \rightarrow 2$ transitions in all pointings. The primary energy levels of both of these molecules are split by hyperfine interactions, giving spectra with more structure than is accessible using Z-Spec. The intensities quoted in Table 3 are obtained by fitting a single Gaussian profile and thus represent the total integrated intensity for all transitions. These species have been detected, for the first time, in M82 by Henkel et al. (1988) and Fuente et al. (2005), but the $J=3 \rightarrow 2$ transition of $\mathrm{C}_{2} \mathrm{H}$ is a new detection. Neither of these authors quotes the intensity seen in all hyperfine components, making a direct comparison difficult. Our emphasis is on a study of physical conditions which, if it included these species, would require knowledge of the intensities of the hyperfine components. Therefore, we do not include the $\mathrm{CN}$ and $\mathrm{C}_{2} \mathrm{H}$ in this analysis that follows.

\subsection{3. $\mathrm{HCO}^{+}, \mathrm{HCN}$, and $\mathrm{HNC}$}

The $J=3 \rightarrow 2$ transitions of the $\mathrm{HCO}^{+}, \mathrm{HCN}$, and $\mathrm{HNC}$ molecules are strongly detected in all three pointings. The $\mathrm{HCO}^{+}$and $\mathrm{HCN}$ transitions have been previously detected by Wild et al. (1992), however, the only previous detection of $\mathrm{HNC}$ in M82 has been the $J=1 \rightarrow 0$ line by Huettemeister et al. (1995). Comparing the $12^{\prime \prime}$ beam measurements from Wild et al. (1992) to ours suggest a beam scaling exponent of roughly -1 , consistent with the continuum and $\mathrm{CO}$ values. We use our measurements and measurements of other transitions in the subsequent analysis.

\subsection{4. $C S$}

The $J=4 \rightarrow 3,5 \rightarrow 4$, and $6 \rightarrow 5$ transitions of CS lie in the Z-Spec band and it's simple ladder of rotational transitions make it an ideal candidate for study with our instrument. Unfortunately, the lines in our band are not very bright in M82 so we can only give upper limits for the $J=6 \rightarrow 5$ lines and the $J=5 \rightarrow 4$ lines in the NE and SW pointings. The transitions we detect have been seen in M82 by Bavet et al. (2008) for the CEN pointing and Bayet et al. (2009) for the NE and SW pointings. Longer integration times should enable the first detections of the $J=6 \rightarrow 5$ lines, particularly in the CEN pointing where the fitted intensity is $2.7 \sigma$. As with $\mathrm{HCO}^{+}, \mathrm{HCN}$, and $\mathrm{HNC}$, we combine our measurements with the other measured transitions for the analysis that follows.

\subsection{5. $\mathrm{H}_{2} \mathrm{CO}$}

We have identified the $3_{03} \rightarrow 2_{02}, 3_{13} \rightarrow 2_{12}, 4_{14} \rightarrow 3_{13}$, $4_{04} \rightarrow 3_{03}$, and $4_{13} \rightarrow 3_{12}$ transitions of formaldehyde $\left(\mathrm{H}_{2} \mathrm{CO}\right)$ which are detected in at least one of the three pointings; the latter four of these transitions have not been detected previously. Two of these transitions, $3_{03} \rightarrow 2_{02}$ and $4_{04} \rightarrow 3_{03}$, may be blended with other higher energy $\mathrm{H}_{2} \mathrm{CO}$ lines and our reported integrated 
intensity should be interpreted as the sum of the intensities of all of these blended lines. The feature at 218 $\mathrm{GHz}$ has been studied with the heterodyne receiver array HERA on the IRAM 30m telescope by Mühle et al. (2007). They detected three formaldehyde transitions, $3_{03} \rightarrow 2_{02}, 3_{22} \rightarrow 2_{21}$, and $3_{21} \rightarrow 2_{20}$, near our $\mathrm{NE}$ and SW pointings along with a possible detection of a methanol line in the NE pointing. In addition, they demonstrated the power of using formaldehyde lines to trace both temperature and density of the molecular gas. Higher-resolution followup of the lines we have identified would provide a powerful extension to their work.

\subsection{6. $\mathrm{CH}_{3} \mathrm{CCH}$}

Methyl acetylene $\left(\mathrm{CH}_{3} \mathrm{CCH}\right)$ has the largest number of atoms of any molecule detected outside our galaxy and several transitions have been seen previously in the SW lobe of M82 (Mauersberger et al. 1991). Our measurements in the NE and CEN pointings as well as all the measurements of the $J=12 \rightarrow 11$ and $J=15 \rightarrow 14$ transitions are new. We suspect that the $J=15 \rightarrow 14$ transition may be contaminated with an unidentified feature that is causing the fitted flux in the SW pointing to be unrealistically high. This spectral feature is right at our three-sigma threshold and additional data are needed to precisely determine the line identifications.

\section{ANALYSIS}

\subsection{Excitation and Radiative Transfer Modeling}

We turn now to a study of the physical conditions in the dense gas, as probed with rotational transitions of $\mathrm{HCN}, \mathrm{HNC}, \mathrm{HCO}^{+}$, and CS. To make useful inferences about the conditions in M82's molecular gas, modeling is required. The approach is to adopt basic input parameters such as total amount of gas in the beam (column density), gas density, temperature, and abundance of the species under consideration and then calculate the resulting line intensities. A grid of such calculations over ranges of input parameters can then provide a framework to interpret the observations. We note that the transitions we study have a range of critical densities ranging from $10^{4}$ to $10^{8} \mathrm{~cm}^{-3}$.

We use the RADEX code (van der Tak et al. 2008) for our excitation and radiative transfer modeling. The primary inputs to RADEX are the choice of molecule, the kinetic temperature of the molecular gas $T_{\text {kin }}$, the density of molecular hydrogen $n_{\mathrm{H}_{2}}$ in $\mathrm{cm}^{-3}$, and the column density of the species $N_{\mathrm{mol}}$ in $\mathrm{cm}^{-2}$. The radiative transfer calculation depends on $N_{\mathrm{mol}} / \Delta v$, where $\Delta v$ is the velocity width of the line. Z-Spec cannot measure the linewidth, so $\Delta v=250 \mathrm{~km} \mathrm{~s}^{-1}$ is used throughout the radiative transfer modeling to be consistent with the linewidth used for the spectral fits. RADEX does not assume local thermal equilibrium, but uses an escape probability formalism that connects the optical depth to the chance an emitted photon escapes the source cloud. Several different physical models for this escape probability have been derived; we use the expanding spherical shell model. However, the results are very insensitive to the choice of escape probability. Starting with an initial guess for the level population distribution, RADEX computes the optical depths of all the molecule's transitions, from which a new level population distribution can be calculated. This process iterates until a self-consistent solution is achieved such that the optical depth changes by less than a default tolerance from one iteration to the next. We use the collisional excitation rates calculated in Lique et al. (2006) and Lique \& Spielfiedel (2007) for the CS species and the rates from the online database outlined in Schöier et al. (2005) for the three remaining species.

\subsection{Parameter Likelihood Estimation}

Calculated line intensities are then compared to the intensity measurements of the species under consideration. An additional area filling factor parameter $\Phi_{\mathrm{A}}$ must be included in the model because the clumps of gas producing the radiation do not in general fill the beam. $\Phi_{\mathrm{A}}$ scales down the line intensities from RADEX so that they can be directly related to the measurements. When $\Phi_{\mathrm{A}}$ is less than $1, N_{\text {mol }}$ represents the column density of an individual radiating clump while the product $\Phi_{\mathrm{A}} N_{\mathrm{mol}}$ is the beam-averaged column density, $\left\langle N_{\text {mol }}>\right.$.

The line-intensity measurements are inherently uncertain and the physical interpretation using models should reflect that. A Bayesian method for calculating likelihood distributions for various physical quantities of interest can be used to address this measurement uncertainty (Ward 2002; Ward et al. 2003 [hereafter W03]). The method constructs the probability distribution of obtaining the measurements with their associated errors given a set of physical parameters, assuming the measurements are independent and the errors are Gaussian distributed. Using a prior-probability density function for the range of physical parameters, the probability distribution of the measurements given the physical parameters can be inverted into a likelihood distribution for the physical parameters given the measurement results.

Bayes' Postulate says that the prior probability density function should be uniform for all cases in the absence of prior knowledge. The prior probability distributions used for this analysis are assumed to be logarithmically uniform in all model parameters. However, this prior probability is used to exclude certain non-physical situations relating to large column densities and small molecular hydrogen densities. Both constraints require knowledge of the molecular abundance ratio, $X_{\mathrm{mol}} \equiv n_{\mathrm{mol}} / n_{\mathrm{H}_{2}}$. These constraints, described in detail by W03, limit the total molecular mass contained in the telescope beam to less than the dynamical mass of the galaxy $\left(2.0 \times 10^{9} M_{\odot}\right.$, based on the estimates given by W03 and Panuzzo et al. (2010) [hereafter P10]) and limit the column length, equal to the column density divided by the number density, to less than the length of the bright molecular emission on the plane of the sky. In addition, models with optical depths larger than 100 in any transition are excluded because very large optical depths are not appropriate for the species under consideration and RADEX is not accurate when the optical depth is this large.

A critical aspect of the analysis is the scaling of the published measurements of various transitions to a common beamsize. The limits of beam scaling are $\theta^{0}$ for a source that fills the beam for all measurements, and $\theta^{-2}$ for a source that is always smaller than the beam. M82 is in an intermediate range with respect to the $25^{\prime \prime}-35^{\prime \prime} \mathrm{Z}$ Spec beam; it is neither fully point-like nor beam-filling. 
Based on the CO and mm-wave continuum maps, we use an intermediate beam scaling of $\theta^{-1}$, appropriate for the distribution which is to first order extended along the major-axis, but unresolved along the minor axis.

\subsection{Multi-Species Model}

Four of the species detected in this survey have the required radiative and collisional rate data available in an online database (Schöier et al.|2005) or in the literature (Lique et al. 2006; Lique \& Spielfiedel 2007): $\mathrm{HCO}^{+}$, HCN, HNC, and CS. The method described in W03 and outlined in the previous section was developed for analyzing 11 transitions of $\mathrm{CO}$ and ${ }^{13} \mathrm{CO}$ and it can be applied to each of the four molecules above individually. However, for three of the four selected molecules, the number of detected transitions (see Table 4) is less than the four primary parameters of the radiative transfer model $\left(T_{\text {kin }}\right.$, $n_{\mathrm{H}_{2}}, N_{\mathrm{mol}}$, and $\left.\Phi_{\mathrm{A}}\right)$. CS and $\mathrm{C}^{34} \mathrm{~S}$ have had several transitions measured but with relatively low signal-to-noise which would lead to very broad constraints from the likelihood analysis.

Observations of these four species in star-forming regions within our Galaxy indicate good correspondence with both the spatial distributions and the line profiles (Brand et al. 2001; Nikolić et al. 2003). High spatial resolution maps of the Galactic circumnuclear disk (Christopher et al. 2005) and of the starburst galaxy NGC 253 (Knudsen et al. 2007) in $\mathrm{HCO}^{+}$and $\mathrm{HCN}$ indicate strong similarity, in general, between the emission of these two molecules.

We thus construct a model in which all four species are characterized by a common kinetic temperature, molecular hydrogen density, molecular hydrogen column density $\left(N_{\mathrm{H}_{2}}\right)$, and filling factor. Each species is modeled with a individual abundance $\left(X_{\mathrm{mol}}\right)$. Extraction of the absolute abundances relative to $\mathrm{H}_{2}$ is not possible with RADEX, but it can constrain the relative abundances of the various species. In the following analysis, CS is chosen as the primary species and the model is parameterized by $T_{\text {kin }}$, $n_{\mathrm{H}_{2}}, N_{\mathrm{CS}}, \Phi_{\mathrm{A}}, X_{\mathrm{HCO}^{+}} / X_{\mathrm{CS}}, X_{\mathrm{HCN}} / X_{\mathrm{CS}}, X_{\mathrm{HNC}} / X_{\mathrm{CS}}$, and $X_{\mathrm{C}^{34} \mathrm{~S}} / X_{\mathrm{CS}}$.

The result of the likelihood analysis is a likelihood matrix with each point in the matrix characterized by a particular value of the four primary species parameters and three secondary species abundance ratios which are used to parameterize the model. As described in W03, likelihood distributions for a single parameter can be obtained by integrating the likelihood matrix along all the other dimensions. These distributions, seen in Figures 3 and 4, can be used to calculate both median values and confidence ranges for the seven parameters in our model (see Table 5). It is also possible to calculate likelihood distributions for parameters which are functions of model parameters, such as the gas pressure, $P=n_{\mathrm{H}_{2}} \times T_{\text {kin }}$, and beam-averaged column density, $\left\langle N_{\mathrm{CS}}\right\rangle=\Phi_{\mathrm{A}} \times N_{\mathrm{CS}}$. The beam-averaged column density can be used to calculate the total molecular mass in the beam by

$$
M_{\text {beam }}=1.5 \times m_{H_{2}} \times \frac{\pi D_{\text {beam }}^{2}}{4} \times \frac{<N_{\text {mol }}>}{X_{\text {mol }}}
$$

where $m_{H_{2}}$ is the mass of a hydrogen molecule, $D_{\text {beam }}$ is the linear diameter of the beam in $\mathrm{cm}$ and the factor of 1.5 accounts for the additional mass of He and dust in the
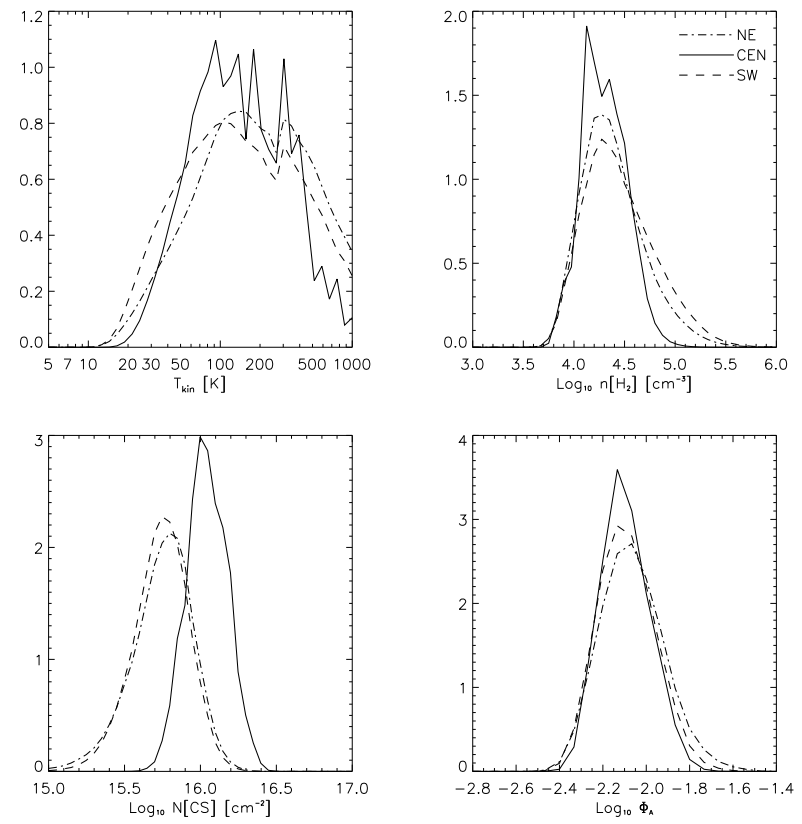

Figure 3. Likelihood distributions for the four primary species parameters, $T_{\mathrm{kin}}, n_{\mathrm{H}_{2}}, N_{\mathrm{CS}}$, and $\Phi_{\mathrm{A}}$, for the five-species radiative transfer likelihood analysis. All of the distributions have well defined peaks, indicating the parameters are well constrained by the measurements within the range of the RADEX simulation. The distributions for the three pointings are plotted in dash-dotted, solid and dashed lines for the NE, CEN, and SW pointings, respectively. The distributions are normalized to have unit integral when integrated over the base-10 logarithm of the parameter value. The jaggedness seen in the $T_{\text {kin }}$ distributions is caused by two factors: first, the spike seen in the distributions for all three pointings at $300 \mathrm{~K}$ is caused by patching together the two sets of colisional rates of CS from Lique et al. (2006) and Lique \& Spielfiedel (2007). The former paper calculated the rates for 31 rotational levels for temperatures up to $300 \mathrm{~K}$ while the later produced rates for 38 rotational and 3 vibrational levels in a higher temperature range. The remaining jaggedness in the CEN pointing $T_{\text {kin }}$ distribution probably comes from a numerical problem in RADEX where, for certain physical conditions, it fails to converge on a stable solution for the optical depth in the CS $J=1 \rightarrow 0$ line. The NE and SW pointings' distributions do not show this effect because they do not have a $\mathrm{CS} J=1 \rightarrow 0$ measurement.

molecular clouds. Distributions for these parameters and their associated two-dimensional distributions are shown in Figure 5 .

The emitting regions containing these molecules are likely to be isolated cores of high-density gas. In that limit, we can estimate the velocity gradient inside the cores with a simple geometrical argument. The characteristic size of an individual core can be estimated by $S_{\text {core }} \approx\left(N_{\mathrm{CS}} / X_{\mathrm{CS}}\right) / n_{\mathrm{H}_{2}}$, which is simply the length defined by the ratio of the molecular hydrogen column and volume densities. This value can be used to estimate the number of cores in the beam by computing the ratio of the area of the emitting region in the beam over the size of a single core, $N_{\text {core }} \approx\left(\Phi_{\mathrm{A}} \times D_{\text {beam }}^{2}\right) / S_{\text {core }}^{2}$. A reasonable approximation for isolated cores where $\Phi_{\mathrm{A}} \ll 1$ is that the observed total line width $\Delta v$ is split up equally among the individual cores. That implies that the velocity gradient in a single core is

$$
\frac{\mathrm{d} v}{\mathrm{~d} r} \approx \frac{\Delta v / N_{\text {core }}}{S_{\text {core }}}=\frac{\Delta v}{D_{\text {beam }}^{2} X_{\mathrm{CS}}} \times \frac{N_{\mathrm{CS}}}{\Phi_{\mathrm{A}} n_{\mathrm{H}_{2}}} .
$$


Table 4

High-Dipole-Moment Species Measurements

\begin{tabular}{|c|c|c|c|c|c|c|c|}
\hline Transition & $\begin{array}{c}\text { Rest Freq. } \\
(\mathrm{GHz})\end{array}$ & $\begin{array}{c}E_{\text {upper }} \\
(\mathrm{K})\end{array}$ & $\begin{array}{l}\text { Obs. Beam } \\
\text { (arcsec) }\end{array}$ & $\begin{array}{c}\text { NE Flux } \\
\left(\mathrm{K} \mathrm{km} \mathrm{s}^{-1}\right)\end{array}$ & $\begin{array}{c}\text { CEN Flux } \\
\left(\mathrm{K} \mathrm{km} \mathrm{s}^{-1}\right)\end{array}$ & $\begin{array}{c}\text { SW Flux } \\
\left(\mathrm{K} \mathrm{km} \mathrm{s}^{-1}\right)\end{array}$ & Refs \\
\hline $\begin{array}{l}J=1 \rightarrow 0 \\
J=2 \rightarrow 1 \\
J=3 \rightarrow 2 \\
J=4 \rightarrow 3 \\
J=5 \rightarrow 4 \\
J=6 \rightarrow 5\end{array}$ & $\begin{array}{c}48.991 \\
97.981 \\
146.969 \\
195.954 \\
244.936 \\
293.912 \\
\end{array}$ & $\begin{array}{c}2.4 \\
7.1 \\
14.1 \\
23.5 \\
35.3 \\
49.4 \\
\end{array}$ & $\begin{array}{c}36 \\
25.1 \\
16.7 \\
37.1 \\
30.8 \\
26.3 \\
\end{array}$ & $\begin{array}{l}\text { CS } \\
\qquad \begin{array}{l}\ldots \\
9.4 \pm 0.2 \\
8.9 \pm 0.1 \\
4.3 \pm 0.8 \\
\quad<0.5 \\
\quad<1.0\end{array}\end{array}$ & $\begin{array}{c}16.2 \pm 1.1 \\
13.3 \pm 0.3 \\
11.2 \pm 0.3 \\
4.4 \pm 0.8 \\
2.3 \pm 0.5 \\
<1.1\end{array}$ & $\begin{array}{l}8.9 \pm 0.2 \\
7.6 \pm 1.7 \\
4.1 \pm 0.8 \\
<0.5 \\
<1.0\end{array}$ & $\begin{array}{l}2 \\
3,4 \\
3,4,5 \\
1 \\
1 \\
1\end{array}$ \\
\hline $\begin{array}{l}J=3 \rightarrow 2 \\
J=4 \rightarrow 3 \\
J=5 \rightarrow 4 \\
J=6 \rightarrow 5\end{array}$ & $\begin{array}{l}144.617 \\
192.818 \\
241.016 \\
289.209 \\
\end{array}$ & $\begin{array}{l}13.9 \\
23.1 \\
34.7 \\
48.6\end{array}$ & $\begin{array}{c}17 \\
37.1 \\
30.8 \\
26.3 \\
\end{array}$ & $\begin{array}{l}\sim 0.5 \pm 50 \% \\
\quad<0.8 \\
\quad<0.5 \\
\quad<1.0\end{array}$ & $\begin{array}{l}0.6 \pm 0.1 \\
\quad<0.8 \\
<0.5 \\
<1.1\end{array}$ & $\begin{aligned} \sim 0.4 & \pm 50 \% \\
& <0.8 \\
& <0.5 \\
& <1.0\end{aligned}$ & $\begin{array}{l}6 \\
1 \\
1 \\
1\end{array}$ \\
\hline $\begin{array}{l}J=1 \rightarrow 0 \\
J=3 \rightarrow 2 \\
J=4 \rightarrow 3\end{array}$ & $\begin{array}{c}89.189 \\
267.558 \\
356.734\end{array}$ & $\begin{array}{c}4.3 \\
25.7 \\
42.8\end{array}$ & $\begin{array}{c}23 \\
28.6 \\
14\end{array}$ & $\begin{array}{l}\mathrm{O}^{+} \\
35.0 \pm 2.0 \\
13.7 \pm 0.8 \\
23.6 \pm 1.7\end{array}$ & $\begin{array}{l}38.3 \pm 2.0 \\
16.9 \pm 0.9 \\
22.2 \pm 1.7\end{array}$ & $\begin{array}{l}37.2 \pm 2.0 \\
15.4 \pm 0.9 \\
22.6 \pm 1.9\end{array}$ & $\begin{array}{l}7 \\
1 \\
8\end{array}$ \\
\hline $\begin{array}{l}J=1 \rightarrow 0 \\
J=3 \rightarrow 2 \\
J=4 \rightarrow 3\end{array}$ & $\begin{array}{c}88.632 \\
265.886 \\
354.505\end{array}$ & $\begin{array}{c}4.3 \\
25.5 \\
42.5\end{array}$ & $\begin{array}{c}23 \\
28.8 \\
14\end{array}$ & $\begin{array}{c}21.8 \pm 2.0 \\
5.1 \pm 0.8 \\
5.6 \pm 0.6\end{array}$ & $\begin{array}{l}18.5 \pm 2.0 \\
7.0 \pm 0.8 \\
9.0 \pm 0.7\end{array}$ & $\begin{array}{l}23.7 \pm 2.0 \\
6.9 \pm 0.8 \\
6.1 \pm 0.4\end{array}$ & $\begin{array}{l}7 \\
1 \\
8\end{array}$ \\
\hline $\begin{array}{l}J=1 \rightarrow 0 \\
J=3 \rightarrow 2\end{array}$ & $\begin{array}{c}90.664 \\
271.981\end{array}$ & $\begin{array}{c}4.4 \\
26.1\end{array}$ & $\begin{array}{c}25 \\
28.2\end{array}$ & $\begin{array}{l}\text { INC } \\
10.7 \pm 2.0 \\
2.4 \pm 0.6\end{array}$ & $\begin{array}{c}13.4 \pm 0.8 \\
2.7 \pm 0.7\end{array}$ & $\begin{array}{c}12.3 \pm 3.0 \\
3.5 \pm 0.6\end{array}$ & $\begin{array}{l}9 \\
1\end{array}$ \\
\hline
\end{tabular}

References. - (1) This Work; (2) Paglione et al. [1995; (3) Bavet et al.|2008; (4) Bavet et al.| 2009; (5) Mauersberger \& Henkel 1989; (6) Martín et al. 2009; (7) Nguven-Q-Rieu et al. 1989; (8) Seaquist \& Fraver 2000; (9) Huettemeister et al. 1995.

Note. - Fluxes and upper limits used in our multi-species radiative transfer likelihood analysis. In addition to the given statistical error, a $10 \%$ calibration error is added in quadrature to each measurements' uncertainty. An additional $10 \%$ error is added to the lines measured in beams smaller than $18^{\prime \prime}$ and to the CS $J=1 \rightarrow 0$ line. The CS $J=1 \rightarrow 0$ line has only been measured for the CEN pointing and thus is not included for the likelihood analysis for the other pointings. The $\mathrm{C}^{34} \mathrm{~S} J=3 \rightarrow 2$ has also only been measured in the CEN pointing; we estimate the flux in the other pointings based on the $J=3 \rightarrow 2$ line ratio between CS and $\mathrm{C}^{34} \mathrm{~S}$ in the CEN pointing and apply a $50 \%$ error to these estimates for the likelihood analysis. The $\mathrm{C}^{34} \mathrm{~S}$ upper limits are based on the uncertainty in the spectral fit for the main-species lines; the actual integrated intensity in the $\mathrm{C}^{34} \mathrm{~S}$ lines is well below the detection threshold of our data.

Table 5

Multi-Species Modeling Results

\begin{tabular}{|c|c|c|c|c|c|c|}
\hline \multirow[t]{2}{*}{ Quantity } & \multicolumn{2}{|c|}{ NE Pointing } & \multicolumn{2}{|c|}{ CEN Pointing } & \multicolumn{2}{|c|}{ SW Pointing } \\
\hline & Median & Range & Median & Range & Median & Range \\
\hline & \multicolumn{6}{|c|}{ Primary Species Parameters } \\
\hline$T_{\text {kin }}(\mathrm{K})$ & 160 & $58-470$ & 130 & $56-320$ & 130 & $44-410$ \\
\hline$n_{\mathrm{H}_{2}}\left(\mathrm{~cm}^{-3}\right)$ & $10^{4.3}$ & $10^{4.0}-10^{4.7}$ & $10^{4.2}$ & $10^{4.0}-10^{4.5}$ & $10^{4.4}$ & $10^{4.1}-10^{4.8}$ \\
\hline$N_{\mathrm{CS}}\left(\mathrm{cm}^{-2}\right)$ & $10^{15.7}$ & $10^{15.5}-10^{15.9}$ & $10^{16.0}$ & $10^{15.9}-10^{16.2}$ & $10^{15.7}$ & $10^{15.5}-10^{15.9}$ \\
\hline$\Phi_{\mathrm{A}}$ & $10^{-2.1}$ & $10^{-2.3}-10^{-1.9}$ & $10^{-2.1}$ & $10^{-2.3}-10^{-2.0}$ & $10^{-2.1}$ & $10^{-2.3}-10^{-2.0}$ \\
\hline \multicolumn{7}{|c|}{ Secondary Species Relative Abundances } \\
\hline$X_{\mathrm{HCO}}+/ X_{\mathrm{CS}}$ & $10^{0.1}$ & $10^{0.0}-10^{0.2}$ & $10^{0.06}$ & $10^{-0.02}-10^{0.13}$ & $10^{0.2}$ & $10^{0.1}-10^{0.3}$ \\
\hline$X_{\mathrm{HCN}} / X_{\mathrm{CS}}$ & $10^{0.4}$ & $10^{0.3}-10^{0.5}$ & $10^{0.3}$ & $10^{0.2}-10^{0.4}$ & $10^{0.5}$ & $10^{0.4}-10^{0.6}$ \\
\hline$X_{\mathrm{HNC}} / X_{\mathrm{CS}}$ & $10^{0.0}$ & $10^{-0.2}-10^{0.1}$ & $10^{0.0}$ & $10^{-0.2}-10^{0.1}$ & $10^{0.2}$ & $10^{0.0}-10^{0.3}$ \\
\hline$X_{\mathrm{C}^{34} \mathrm{~S}} / X_{\mathrm{CS}}$ & $10^{-1.7}$ & $10^{-2.7}-10^{-1.3}$ & $10^{-1.6}$ & $10^{-1.7}-10^{-1.4}$ & $10^{-1.8}$ & $10^{-2.7}-10^{-1.4}$ \\
\hline \multicolumn{7}{|c|}{ Projected Parameters } \\
\hline Pressure $\left(\mathrm{K} \mathrm{cm}^{-3}\right)$ & $10^{6.6}$ & $10^{6.3}-10^{6.8}$ & $10^{6.4}$ & $10^{6.2}-10^{6.6}$ & $10^{6.5}$ & $10^{6.3}-10^{6.8}$ \\
\hline$\left\langle N_{\mathrm{CS}}>\left(\mathrm{cm}^{-2}\right)\right.$ & $10^{13.6}$ & $10^{13.5}-10^{13.8}$ & $10^{13.9}$ & $10^{13.8}-10^{14.0}$ & $10^{13.6}$ & $10^{13.4}-10^{13.7}$ \\
\hline $\mathrm{d} v / \mathrm{d} r\left(\mathrm{~km} \mathrm{~s}^{-1} \mathrm{pc}^{-1}\right)$ & 1.9 & $0.6-4.4$ & 4.5 & $3.0-7.2$ & 1.6 & $0.5-4.1$ \\
\hline Total Gas Mass in Beam $\left(M_{\odot}\right)$ & $10^{7.9}$ & $10^{7.8}-10^{8.1}$ & $10^{8.2}$ & $10^{8.1}-10^{8.3}$ & $10^{7.9}$ & $10^{7.8}-10^{8.0}$ \\
\hline
\end{tabular}

Note. - Results obtained from the multiple species radiative transfer modeling of the lines of $\mathrm{CS} \mathrm{HCO}^{+}, \mathrm{HCN}, \mathrm{HNC}$ and $\mathrm{C}^{34} \mathrm{~S}$. The median and $68 \%(1 \sigma)$ confidence ranges are obtained from the likelihood distributions shown in Figures 3 - 5 


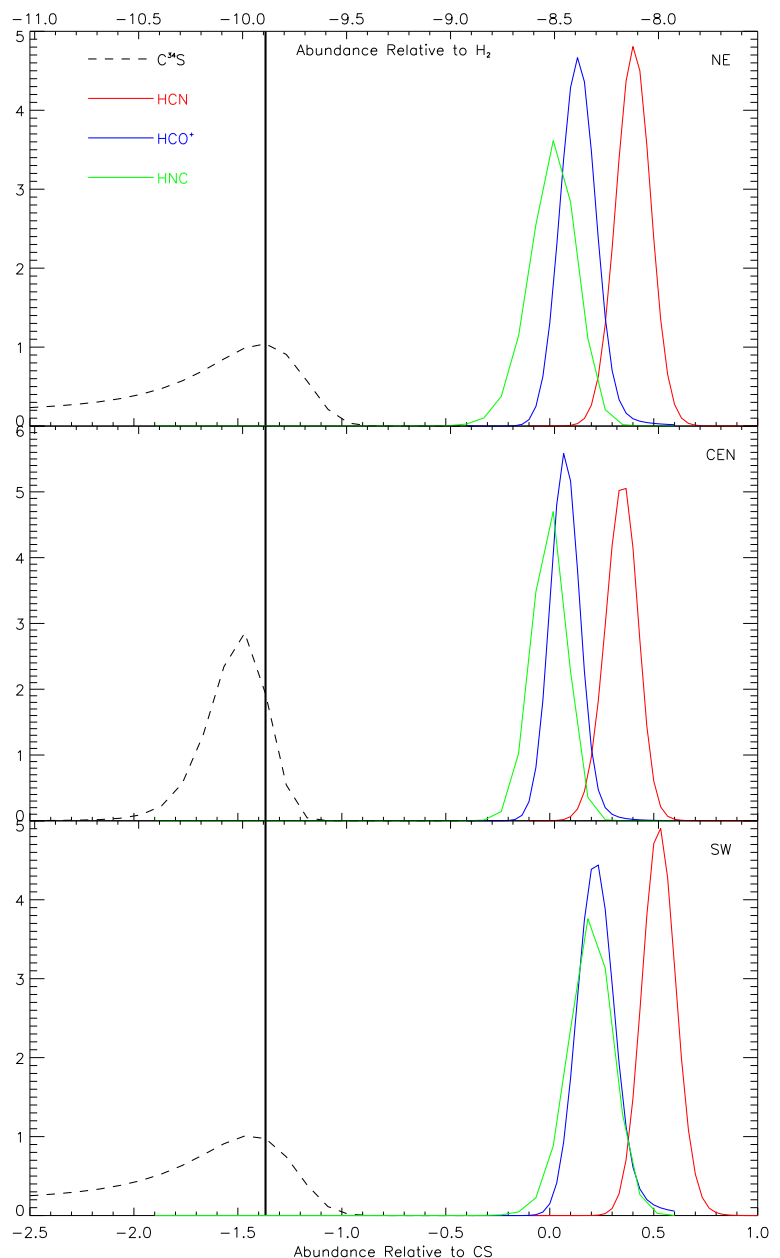

Figure 4. Likelihood distributions for the abundances of the secondary species, $\mathrm{HCO}^{+}, \mathrm{HCN}, \mathrm{HNC}$, and $\mathrm{C}^{34} \mathrm{~S}$ in blue, red, green, and dashed black, respectively, for the NE (top), CEN (middle), and SW (bottom) pointings. The thick vertical black line indicates the solar abundance of ${ }^{34} \mathrm{~S} /{ }^{32} \mathrm{~S}=0.043$ that is assumed for calculating all other likelihood distributions. The bottom axis is labeled with the abundance relative to CS, which is the parameterization used in the model. The top axis shows the abundance relative to $\mathrm{H}_{2}$ using the adopted value of $X_{\mathrm{CS}}=3 \times 10^{-9}$.

The distributions for the core velocity gradient are shown in Figure 5]along with two-dimensional projections of the three-dimensional distributions used for the calculation.

\subsection{Molecular Abundances}

The likelihood distributions of the $\mathrm{HCO}^{+}, \mathrm{HCN}, \mathrm{HNC}$, and $\mathrm{C}^{34} \mathrm{~S}$ abundances relative to that of $\mathrm{CS}$ are shown in Figure 4. These represent the first statistically rigorous measurements of molecular abundances in M82. Average values for the entire starburst nucleus are presented in Table 6. As discussed in Section 4.2, the abundance of CS is used in the likelihood calculation to apply certain physical limits on the parameter space of the radiative transfer grid. We adopt the CS abundance $X_{\mathrm{CS}}$ of $3 \times 10^{-9}$ calculated in Mauersberger \& Henkel (1989). Their calculation is based on assuming optically thin $\mathrm{CS}$ emission and the $\mathrm{CO}$ intensity to $\mathrm{H}_{2}$ column density conversion factor that is observed in the Milky Way. Changing the CS abundance by half an order of mag-
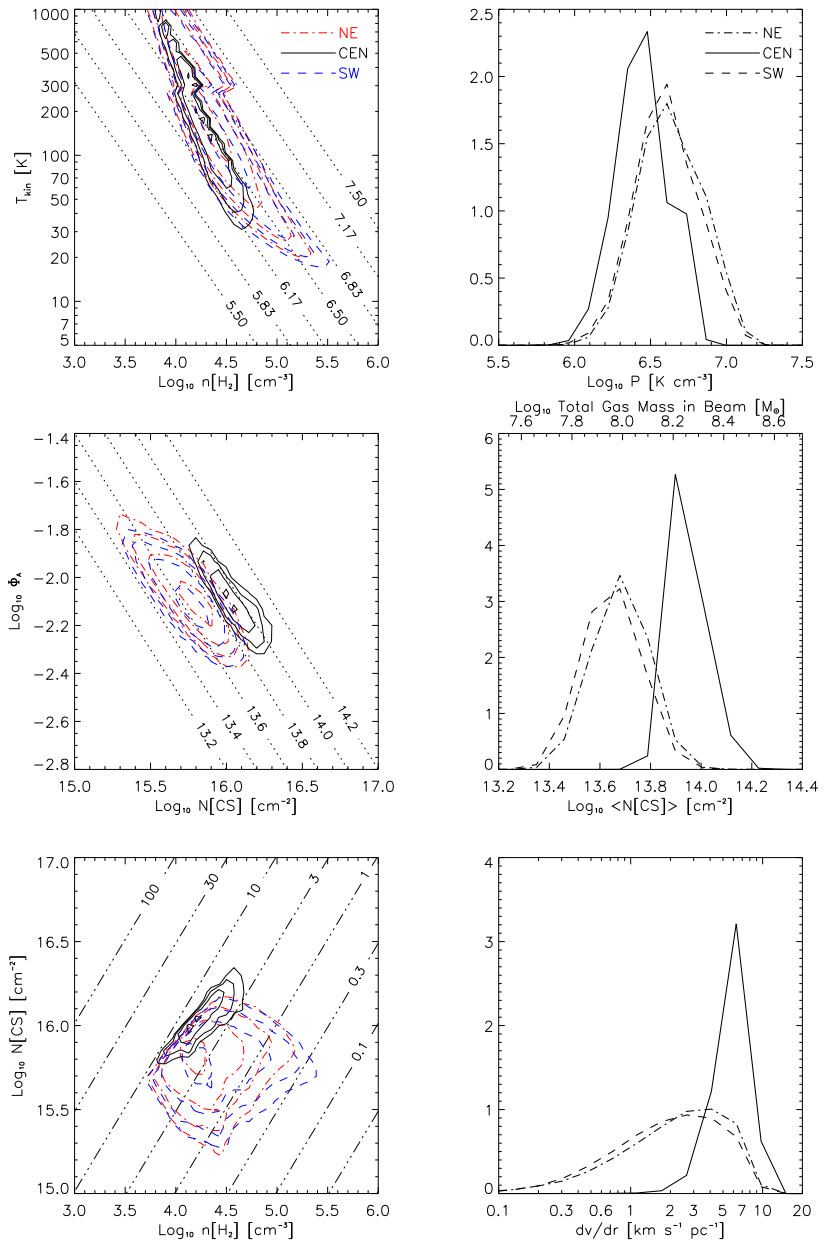

Figure 5. The plots in the left column show two-dimensional likelihood distributions for three pairs of primary species parameters while the right column plots show distributions for parameters that are projected from the corresponding $2 \mathrm{D}$ distributions. The three pointings, NE, CEN, and SW, are indicated by red, black, and blue contour lines in the 2D distributions and with dash-dotted, solid, and dashed lines in the projected $1 \mathrm{D}$ distributions. The contour lines represent $10 \%, 20 \%, 40 \%$, and $80 \%$ of the peak of the $2 \mathrm{D}$ likelihood distributions. The top-left plot has dashed lines of constant pressure, labeled in units of $\log _{10} \mathrm{~K} \mathrm{~cm}{ }^{-3}$; the $T_{\text {kin }}$ versus $n_{\mathrm{H}_{2}}$ distributions are used to create the pressure distributions shown in the top-right plot. Dashed lines of constant beam-averaged column density in the left-middle $\Phi_{\mathrm{A}}$ versus $N_{\mathrm{CS}}$ plot show how the $\left\langle N_{\mathrm{CS}}\right\rangle$ distributions in the right-middle plot are obtained. The alternative axis in the beam-averaged column density plot shows the molecular mass in the beam assuming $X_{\mathrm{CS}}=3 \times 10^{-9}$. The bottom-left plot shows the $N_{\mathrm{CS}}$ versus $n_{\mathrm{H}_{2}}$ distributions; the diagonal dash-dot-dot-dot lines indicate constant velocity gradient in units of $\mathrm{km} \mathrm{s}^{-1} \mathrm{pc}^{-1}$ at the most-likely value of $\Phi_{\mathrm{A}}$. The velocity gradient distributions shown in the bottom-right panel are computed from the three-dimensional distributions of $N_{\mathrm{CS}}, n_{\mathrm{H}_{2}}$, and $\Phi_{\mathrm{A}}$.

nitude either up or down does not affect the likelihood distributions for nearly all of the parameters; the molecular hydrogen density and kinetic temperature distributions shift down and up, respectively, with increasing CS abundance such that the distribution of gas pressure is relatively unchanged. The agreement between the measured and most-likely model's integrated line intensities, shown graphically in Figure 6 for $X_{\mathrm{CS}}=3 \times 10^{-9}$, is not significantly impacted by changing $X_{\mathrm{CS}}$. Furthermore, 
Table 6

Molecular Abundance Ratios for M82

\begin{tabular}{|c|c|c|c|c|c|}
\hline Species & CS & $\mathrm{HCO}^{+}$ & $\mathrm{HCN}$ & $\mathrm{HNC}$ & $\mathrm{C}^{34} \mathrm{~S}$ \\
\hline $\log _{10}\left(X_{\mathrm{mol}}\right)$ & -8.5 & -8.4 & -8.1 & -8.5 & -9.9 \\
\hline $\begin{array}{l}\text { Note. - A } \\
\text { rived from the } \\
\text { abundance of } \\
\text { to CS and the } \\
\text { Mauersberger } 8 \\
\text { four modeled a } \\
\text { for CS in logar }\end{array}$ & $\begin{array}{l}\text { latip } \\
\text { bund } \\
\text { bund }\end{array}$ & $\begin{array}{l}\text { specie } \\
\text { HCN, } \\
\text { ice for } \\
11989\end{array}$ & $\begin{array}{l}\text { aodeli } \\
\text { vC, an } \\
\text { S rela } \\
\text { The u }\end{array}$ & 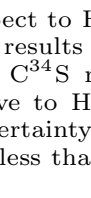 & $\begin{array}{l}\text { ) de- } \\
\text { or the } \\
\text { lative } \\
\text { from } \\
\text { n the } \\
\pm 0.5\end{array}$ \\
\hline
\end{tabular}

while the likelihood distributions for the $\mathrm{C}^{34} \mathrm{~S}$ abundance show slight differences between the three pointings, we have little reason to suspect that this value would be much different from the solar isotopic abundance ratio of ${ }^{34} \mathrm{~S} /{ }^{32} \mathrm{~S}=0.043$. Therefore, we impose this isotopic ratio for the other likelihood calculations.

Of course, changing the assumed CS abundance impacts the conversion from the relative abundance ratios to standard abundances relative to $\mathrm{H}_{2}$. Also, while the beam-averaged column density distribution does not change when using different values for the CS abundance, the conversion from $\left\langle N_{\mathrm{CS}}\right\rangle$ to total mass is inversely proportional to the abundance (see equation 44). Of the three values of CS abundance we have used, the value from Mauersberger \& Henkel (1989) seems to be the most reasonable; lowering it drives the total molecular mass too high and raising it increases the temperatures to extreme values.

While our likelihood analysis is an independent confirmation of the CS abundance estimated by Mauersberger \& Henkel (1989), it should be noted that there is significant debate about the $\mathrm{CO}$ intensity $\left(I_{\mathrm{CO}}\right)$ to molecular hydrogen column density $\left(N_{\mathrm{H}_{2}}\right)$ conversion factor, $\mathcal{X}_{\mathrm{CO}} \equiv N_{\mathrm{H}_{2}} / I_{\mathrm{CO}}$, used in their calculation of CS abundance. Their value was $2.2-2.5$ times larger than more recent measurements of $\mathcal{X}_{\mathrm{CO}}$ in the Milky Way $\left(1.6 \times 10^{20} \mathrm{~cm}^{-2}\left(\mathrm{~K} \mathrm{~km} \mathrm{~s}^{-1}\right)^{-1}\right.$, Hunter et al. 1997; $1.8 \times 10^{20} \mathrm{~cm}^{-2}\left(\mathrm{~K} \mathrm{~km} \mathrm{~s}^{-1}\right)^{-1}$, Dame et al. 2001). Furthermore, a detailed analysis of an interferometric $\mathrm{CO}$ $J=1 \rightarrow 0$ map of the nucleus of M82 showed that $\mathcal{X}_{\mathrm{CO}}$ is roughly 2-10 times less than what is measured in the Milky Way (Weiß et al. 2001). Quantitative modeling of ensembles of unresolved giant molecular clouds by Maloney \& Black (1988) showed that $\mathcal{X}_{\mathrm{CO}}$ is proportional to $T_{\mathrm{kin}}^{-1} n_{\mathrm{H}_{2}}^{1 / 2}$; this proportionality was confirmed in M82 by Weiß et al. (2001). Studies of several galaxies have also shown that $\mathcal{X}_{\mathrm{CO}}$ also depends on metalicity (Wilson 1995; Boselli et al. 2002) and CO intensity (Nakai \& Kuno 1995). The deviations from the standard Milky Way value can be significant; Casasola et al. (2007) measured the conversion factor in several giant molecular associations in M81, the interaction partner of M82 and NGC 3077 (Yun et al. 1994), and found the galactic average of $\mathcal{X}_{\mathrm{CO}}$ to be 20 times larger than the Milky way value. In spite of these developments in the understanding of $\mathcal{X}_{\mathrm{CO}}$ since the publication of Mauersberger \& Henkel (1989), our results indicate that their estimate for the CS abundance is correct to within a half an order of magnitude.

Adopting the value $X_{\mathrm{CS}}=3 \times 10^{-9}$ allows for compar- ison of our abundance measurements to those found in the literature. For the most part, M82 is assumed to have abundances similar to those found in regions of high-mass star formation in our Galaxy such as Sgr B2 or Orion. Wild et al. (1992) quote $X_{\mathrm{HCO}^{+}}=1 \times 10^{-8}$ and $X_{\mathrm{HCN}}=$ $2 \times 10^{-8}$ which have also been used by other authors (e.g., Seaquist \& Fraver 2000). These are somewhat higher than what we measure though we agree that HCN is twice as abundant as $\mathrm{HCO}^{+}$. Huettemeister et al. (1995) use their measurements of $\mathrm{HCN} J=1 \rightarrow 0$ and the CO intensity to $\mathrm{H}_{2}$ column density conversion factor to calculate $X_{\mathrm{HNC}}=1.4 \times 10^{-10}$, assuming the $\mathrm{HCN}$ emission is optically thin. Our measurements of the HNC $J=3 \rightarrow 2$ contradict the optically-thin assumption and our models point to an abundance 1.5 orders of magnitude larger, roughly equal to the CS abundance.

\subsection{Mass of Dense Gas}

The total mass of molecular gas in the nuclear region can be estimated from the total mass in the beam measured for each of the three pointings by adopting a particular geometry for the central region. If the area of the nucleus is $A_{\mathrm{n}}$ and the area of overlap between our $30^{\prime \prime}$ beam and this nuclear area is $A_{\mathrm{ol}}$, then the total mass in the nucleus can be calculated using

$$
\begin{aligned}
M_{\mathrm{total}}= & M_{\mathrm{CEN}}+M_{\mathrm{NE}}\left(\frac{A_{\mathrm{n}}-A_{\mathrm{ol}}}{2 A_{\mathrm{ol}}}\right) \\
& +M_{\mathrm{SW}}\left(\frac{A_{\mathrm{n}}-A_{\mathrm{ol}}}{2 A_{\mathrm{ol}}}\right),
\end{aligned}
$$

where $M_{\mathrm{CEN}}, M_{\mathrm{NE}}$, and $M_{\mathrm{SW}}$ are the masses measured in the three pointings. This equation uses the mass surface density implied by $M_{\mathrm{NE}}$ and $M_{\mathrm{SW}}$ to account for the mass not covered on the left and right sides of the CEN pointing. If we adopt a rectangular nuclear geometry $50^{\prime \prime}$ $\times 15^{\prime \prime}$, as indicated by the CO $J=2 \rightarrow 1$ interferometer maps in Weiß et al. (2001), then the area scaling factor $\left(A_{\mathrm{n}}-A_{\mathrm{ol}}\right) / 2 A_{\mathrm{ol}}=0.37$ and $M_{\text {total }}=2.2 \pm 0.5 \times 10^{8} M_{\odot}$ in the nuclear starburst. This value is comparable to the total mass traced in CO from both multi-line studies (W03; $2.0 \times 10^{8} M_{\odot}$ when corrected to $\left.d=3.86 \mathrm{Mpc}\right)$ and via interferometric low-J $\mathrm{CO}$ and ${ }^{13} \mathrm{CO}$ imaging (Weiß et al. 2001).

Since we measure a gas mass with a suite of transitions, we can provide a calibration of the HCN $\mathcal{X}$ factor, $\mathcal{X}_{\mathrm{HCN}}$, which converts $\mathrm{HCN}$ line luminosity (in temperature units) to mass of dense gas. Assuming that the source couples to the various beam sizes as $\theta^{1}$, we find $\mathcal{X}_{\mathrm{HCN}, J=1}$ of $10-15$, in agreement with the canonical value of 10 derived from virial considerations by Gao \& Solomon (2004a,b). Since HCN is sub-thermally excited, using the $J=3 \rightarrow 2$ transition $\mathcal{X}_{\mathrm{HCN}}$ is much higher with values of $36-65$.

\section{DISCUSSION}

\subsection{Physical Conditions and Relationship to CO-Traced and Atomic Gas}

Our likelihoods suggest temperatures between 50 $500 \mathrm{~K}$, broadly consistent with the warm components modeled by W03, up to $J=6 \rightarrow 5$, and somewhat lower than the $400-800 \mathrm{~K}$ derived by $\mathrm{P} 10$ in considering all 


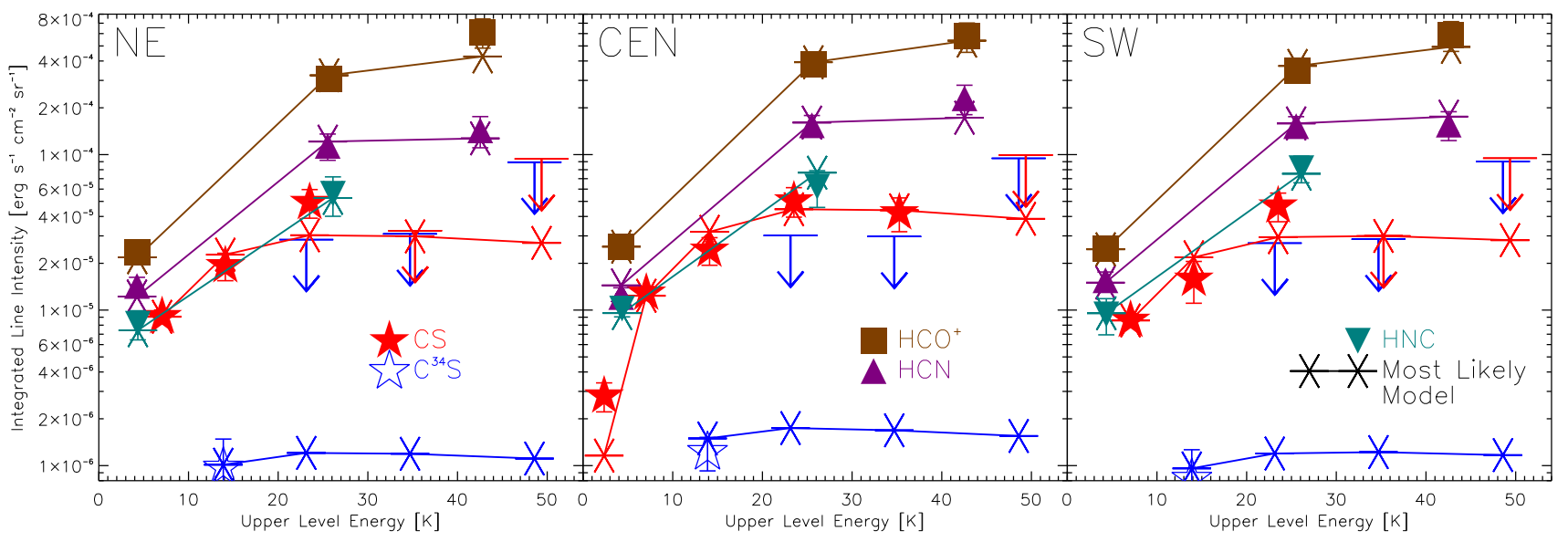

Figure 6. Measured and modeled spectral energy distributions for the five molecules of the multi-species radiative transfer likelihood analysis for the three pointings, NE (left), CEN (center), and SW(right). The measurements of CS, $\mathrm{HCO}^{+}, \mathrm{HCN}^{\mathrm{H}} \mathrm{HNC}$, and $\mathrm{C}^{34} \mathrm{~S}$, from Table 4 scaled to a common $30^{\prime \prime}$ beam, are marked with red stars, brown squares, purple triangles, teal triangles and open blue stars, respectively. The error bars include both measurement and adopted calibration error, if any. Three-sigma upper limits are indicated with downward arrows using the same color scheme as the measurements. The most-likely model from the likelihood analysis is indicated by the six-pointed stars connected by lines, in colors to match the measurements.

of the transitions up to $J=13 \rightarrow 12$ as measured with Herschel SPIRE. Our results are also consistent with the temperature of $200 \mathrm{~K}$ inferred from the formaldehyde measurements and analysis of Mühle et al. (2007). The relatively low precision with which we measure the temperature is not surprising since the most likely temperatures are generally higher than the upper level energies of the transitions we are studying (e.g., $\mathrm{HCN} J=3: T=25$ $\mathrm{K})$. We do note that our results do not support the presence of substantial amounts of gas at temperatures below $\sim 30 \mathrm{~K}$ unless the density is so low that the transitions in our analysis would not be excited. This means that the drop in line intensity with $J$ (in temperature units) is due to sub-thermal excitation of the levels above $J=1$, and the run of line intensity with $J$ should thus provide a reliable density measurement. Indeed, our derived median densities are $1.5-3 \times 10^{4} \mathrm{~cm}^{-3}$, sub-critical for the transitions above $200 \mathrm{GHz}$.

Our derived densities are larger than those derived with CO studies. W03 find $600-6000 \mathrm{~cm}^{-3}$ and P10 find 1000-13000, though we do note in some cases W03 find densities poorly constrained on the high-density end. The product of temperature and density is the thermal pressure, and we find values of $1-4 \times 10^{6} \mathrm{~K} \mathrm{~cm}^{-3}$, an order of magnitude higher than the pressure inferred by W03 $\left(0.5-4 \times 10^{5} \mathrm{~K} \mathrm{~cm}^{-3}\right)$, but comparable to that derived by P10 using all of the CO transitions. Our high densities may reflect the fact that we are probing preferentially high-density cores, in approximate pressure equilibrium with the larger, more diffuse envelopes which produce the bulk of the CO. This should not be surprising since HCN, $\mathrm{HNC}$, and CS are generally found in UV-shielded cores as their dissociation energies are less than that of $\mathrm{CO}$, and they don't have generally achieve sufficient column densities to self-shield. For instance, the photo-dissociation region (PDR) chemical models of Fuente et al. (2008) show that $\mathrm{HCN}$ exists primarily within $A_{\mathrm{V}}>5$.

We compare our results with the studies of the photo-dissociated atomic gas. Kaufman et al. (1999) and Colbert et al. (1999) have applied a PDR model (updated from Wolfire et al. 1990; Tielens \& Hollenbach 1985]5 to [C II] and [O I] fine-structure-line measurements from the Kuiper Airborne Observatory (KAO) and ISO Long-wavelength Spectrometer (LWS), respectively. PDR conditions are parametrized in terms of the density and UV field strength $G_{0}$. Estimates for the M82 central starburst range from: 1) $n_{\mathrm{H}_{2}}=10^{4} \mathrm{~cm}^{-3}$, $G_{0}=10^{3.5}$ (Kaufman et al. 1999, assuming the [C II] emission is uniformly distributed over its $55^{\prime \prime}$ beam, so only a small fraction arises in the [O I]-emitting region), 2) $n_{\mathrm{H}_{2}}=10^{2.7} \mathrm{~cm}^{-3}, G_{0}=10^{2.5}$ (Kaufman et al. 1999, assuming that all the large-beam [C II] emission arises in the same region as the $[\mathrm{O} \mathrm{I}])$, and 3) $n_{\mathrm{H}_{2}}=10^{3.3} \mathrm{~cm}^{-3}$, $G_{0}=10^{2.8}$ Colbert et al. 1999, using large-beam ISO fluxes, but removing a [C II] contribution from the ionized gas). These estimates form a locus in the $n_{\mathrm{H}_{2}}$, $G_{0}$ plane, with the upper end of the density range becoming consistent with our density likelihood. The UVilluminated surfaces of clouds might be expected to have somewhat lower density than the UV-shielded cores, and we note that with the modeled surface temperatures of $\sim 300 \mathrm{~K}$, the PDR thermal pressures range from $10^{5.3}$ to $10^{6.5}$, broadly consistent with the values derived from the $\mathrm{CO}$ as well as our analysis.

However, the PDR models which fit the atomic line fluxes cannot explain the strength of the mid- $J$ CO transitions. For the range of PDR conditions inferred from the atomic lines, the modeled $\mathrm{CO} J=7 \rightarrow 6$ to $[\mathrm{C} \mathrm{II}]$ intensity ratio is at most $\sim 1.7 \times 10^{-3}$ (at $\left.n=10^{4} \mathrm{~cm}^{-3}\right)$. The CO spectrum indeed peaks (in energy units) at $J=7 \rightarrow 6$ per the Herschel SPIRE measurements (P10). This transition carries a fraction $1.4-2.3 \%$ of the [C II], depending on whether the $[\mathrm{C} \mathrm{II}]$ is resolved or unresolved in its $55^{\prime \prime}$ beam, relative to the $43^{\prime \prime} \mathrm{CO}$ analysis region. Thus the $\mathrm{CO}$ emission in M82 exceeds the

${ }^{5}$ see also http://dustem.astro.umd.edu/pdrt/index.html 
PDR predictions for the measured densities by an order of magnitude. Moreover, this is a lower limit-if the $[\mathrm{C}$ II] includes a contribution from ionized gas (as assumed by Colbert et al. 1999), then the inferred PDR line ratio is even larger, and less consistent with the model. Higher-density PDR models $n>10^{4.5} \mathrm{~cm}^{-2}$ can bring the mid- $J$ CO to $[\mathrm{C} \mathrm{II}]$ into agreement with the observations, but then the $[\mathrm{O} \mathrm{I}]$ transition is over-predicted relative to the observations by a factors of 3-10. Moreover, such high-densities for the PDR would be inconsistent with our measured density likelihood which excludes $n>10^{4.5} \mathrm{~cm}^{-3}$.

Empirically, the suite of [C II], [O I], and mid- $J$ CO emission in M82 does not compare with the PDRs associated with Galactic star-formation regions. In M17, the bright mid- $J$ CO and its widespread distribution with [C II] over several parsecs as viewed in the edgeon PDR led Stutzki et al. (1988); Harris et al. (1987) to a clumpy PDR interpretation. However, their data indicate that the $\mathrm{CO} J=7 \rightarrow 6$ in this source only amounts to some $0.5^{-1} \times 10^{-3}$ relative to the [C II], much less than in M82. The Orion PDR is more extreme, with $\mathrm{CO}$ $J=7 \rightarrow 6$ some $8 \%$ of the [C II] (Schmidt-Burgk 1990; Stacey et al. 1993), but in this case the [O I] $63 \mu \mathrm{m}$ line dominates the PDR line emission with $11 \times$ the [C II] (see Herrmann et al. 1997), so the CO $J=7 \rightarrow 6$ is $\sim 0.8 \%$ of the atomic line emission. While the partitioning of energy between the atomic and warm molecular components in the Orion bar is thus similar to what we observe in M82, we stress again that the line ratio are not a good match, particularly the $[\mathrm{O} \mathrm{I}]$ to $[\mathrm{C} \mathrm{II}]$, which is only $1-$ 1.5 in M82. The bright CO and [O I] in Orion is consistent with a clumpy PDR model which includes clumps with density as high as $10^{7} \mathrm{~cm}^{-3}$ (Burton et al. 1990; Koester et al. 1994; Meixner \& Tielens 1993). Such densities cannot be commonplace in M82 based on line ratios in the atomic gas, the CO analyses, and our analysis of the high-dipole-moment species which indicate typical densities less than $10^{4.5} \mathrm{~cm}^{-3}$.

\subsection{Heating of the Gas}

The poor match to the Galactic PDRs and the inability of the PDR models to explain the powerful mid- $J$ $\mathrm{CO}$ emission suggests that non-UV heating sources may be dominating the energetics of the molecular material in this starburst nucleus. X-rays can be a powerful source of energy input to the gas, producing luminous X-ray Dissociation Regions (XDRs, Maloney et al. 1996). However, the hard X-ray luminosity of M82 is only $1.1 \times 10^{6} L_{\odot}$ (Strickland \& Heckman 2007), completely inadequate to power the observed $\mathrm{CO}$ emission. Moreover, multiple chemical / excitation studies show that the line emission from M82 is not consistent with X-rays being a dominant heating term. The XDR models of Meijerink \& Spaans (2005) and Meijerink et al. (2007) predict more [O I] than is observed (Colbert et al. 1999) at our measured densities around $10^{4} \mathrm{~cm}^{-3}$. Fuente et al. (2008) studied $\mathrm{HOC}^{+}$and $\mathrm{HCO}^{+}$in detail and showed that the $\mathrm{HOC}^{+}$emission is not spatially correlated with X-ray emission, and the line ratios among these species as well as $\mathrm{CO}^{+}$and $\mathrm{CN}$ are well-matched with PDR models with $n>10^{4} \mathrm{~cm}^{-3}$ and $G_{0}=10^{4}$, similar to the values derived in analysis of the atomic lines. They did not consider the total energy budget of the molecular gas, and did not discuss heating mechanisms.

Simiarly, Loenen et al. (2008) have considered XDR models and PDR models with and without extra bulk molecular heating and have predicted line ratios among the $\mathrm{HCN}, \mathrm{HCO}^{+}$, and $\mathrm{HNC} J=1 \rightarrow 0$ transitions. According to their models, the measurements for M82 (Table 4) are inconsistent with XDRs, as XDRs are predicted to have HNC comparable to or brighter than HCN. The line ratios are best fit with PDRs with $n \sim 10^{4.5} \mathrm{~cm}^{-3}$ (their lowest density considered), but with additional bulk heating on the order of $3 \times 10^{-19} \mathrm{erg} \mathrm{s}^{-1} \mathrm{~cm}^{-3}$, $\sim 1 L_{\odot} / M_{\odot}$ input into the molecular material (they ascribe this to mechanical heating).

We thus look for other potential bulk heating sources other than X-rays. Suchkov et al. (1993) proposed that the elevated level of cosmic rays due to the supernova rate in M82 will heat the gas, and their derived cosmic ray enhancement factor relative to the Galaxy of $\sim 500$ has been confirmed by recent measurements of high-energy gamma-rays in M82 (VERITAS Collaboration et al. 2009). Bradford et al. (2003) showed that the energy input from a similar cosmic ray density is indeed well-matched to the total molecular gas cooling in NGC 253 as extrapolated from the $\mathrm{CO}$ transitions up to $J=7 \rightarrow 6$, where the $\mathrm{CO}$ emission appears to peak, (Hailey-Dunsheath et al. 2008). Because the CO excitation and total cooling in M82 are similar (from W03, P10 fluxes), we conclude that cosmic rays are a plausible means of heating the gas.

Another important heating term for bulk molecular material is the dissipation of turbulence (Falgarone \& Puget 1995; Mac Low 1999; Pan \& Padoan 2009; Bradford et al. 2005). The average heating per gram is given by dimensional analysis $0.5 \times \sqrt{3} \sigma_{v}^{3} / L$, where $L$ is the driving scale for the turbulence and $\sigma_{v}$ is the (1-D) RMS velocity spread on this size scale. Pan \& Padoan (2009) conclude that turbulent dissipation with $\sigma_{v} \sim 2.5 \mathrm{~km} \mathrm{~s}^{-1}$ on $0.1-1 \mathrm{pc}$ scales is the dominant heating term on average in Galactic clouds (exceeding cosmic ray heating by factors of $3-4$ ), and that it produces temperatures of $13-36 \mathrm{~K}$ in Galactic clouds with densities of $10^{4}-10^{5} \mathrm{~cm}^{-3}$. For gas densities above $10^{6} \mathrm{~cm}^{-3}$, gas-grain cooling becomes dominant, and limits the increase in the gas temperature, as the dust energy balance is largely independent of the gas temperature. A lower bound to the total cooling per mass in M82 is given by the total CO luminosity in P10 ratioed to the mass derived by W03 using the mid- $J$ lines, $\sim 0.1 L_{\odot} / M_{\odot}$. Achieving this via turbulent dissipation requires $\sigma_{v} \sim 5-20 \mathrm{~km} \mathrm{~s}^{-1}$ on the same $0.1-1$ pc scales. The velocity gradient implied by our radiative transfer analysis of $4-10 \mathrm{~km} \mathrm{~s}^{-1} \mathrm{pc}^{-1}$ is a bit lower than this would suggest, but it becomes consistent if the turbulence is distributed on few-pc scales (e.g., $\sigma_{v} \sim 20 \mathrm{kms}^{-1}, L \sim 5 \mathrm{pc}$ ), as might be expected if it is produced by winds and supernova shocks from young star clusters.

\subsection{Comparison with Expanding Shell Starburst Models}

Our findings are largely consistent with the evolving starburst model of Yao et al. (2006) and Yao (2009), in 
which the gas in the nucleus of M82 is a superposition of expanding spherical bubbles around stellar clusters. The bubble interiors are ionized gas ([H II] regions), and the shells are swept-up molecular gas, so the inside edges are PDR fronts. The PDR surfaces are responsible for most of the atomic line emission, and would agree with the Fuente et al. (2008) results. The PDR shell also generates most of the excited $\mathrm{CO}$ emission, though their model includes mechanical energy input from the shock due to the expansion, which may be a substantial term in the heating of the warm molecular gas.

Model intensities for the high-dipole moment species are not presented, preventing a detailed comparison with our data, but (not surprisingly) the total mass and physical conditions in their modeled shells are similar to what we find with our likelihood analysis. Their estimated gas mass of $2 \times 10^{8} M_{\odot}$ from $\mathrm{CO}$ in the central $1 \mathrm{kpc}$ along the major axis (nearly identical to our modeled $50^{\prime \prime} \times 15^{\prime \prime}$ region) is comparable to our measured $2.2 \times 10^{8} M_{\odot}$, and the shell density at the putative $3-10 \mathrm{Myr}$ age is modeled to be $1-3 \times 10^{4} \mathrm{~cm}^{-3}$, similar to what we find. However, as Yao (2009) notes, there are some inconsistencies in the model. While all of the observed molecular and atomic line emission is reproduced, the stellar luminosity which is required is only $\sim 5 \%$ of the observed far-IR luminosity in the same region, potentially the result of assuming zero pressure for the ambient ISM which results in more mass swept up in the modeled shells than is physical.

\subsection{Warm Star-Forming Gas}

Regardless of the details, the Yao (2009) model is representative of the likely physical situation: a new stellar cluster subjects the surrounding molecular gas to both UV photon and mechanical energy input which heats and compresses it, at least in the first $10 \mathrm{Myr}$ after the starburst. The result is a molecular ISM that is demonstrably warmer than the Galactic cloud cores. Does this mean that the SF is quenched? Fuente et al. (2008) compare line ratios of ions $\left(\mathrm{HOC}^{+}, \mathrm{CO}^{+}\right.$to $\left.\mathrm{HCN}\right)$ with their PDR chemistry model to estimate the total depth of the PDR (ions except $\mathrm{HCO}^{+}$quickly become less abundant with increasing $A_{\mathrm{V}}$ ). Fitting the line ratios to two components, they find some $\sim 87 \%$ of the molecular gas is in small clouds with $A_{\mathrm{V}} \sim 5$ (but large enough to house $\mathrm{HCN}, \mathrm{HNC}$ ) with only $\sim 13 \%$ in clouds with $A_{\mathrm{V}} \sim 50$ and conclude that in general the molecular gas is highly fragmented with clouds too small to form massive stars. Förster Schreiber et al. (2003) found that the SF in M82 has occurred in two bursts, one in the center some $10 \mathrm{Myr}$ ago and one in a circumnuclear ring 5 Myr ago, and that each burst was self-quenching with a timescale of a few Myr due to mechanical energy input into the gas. The total mass of stars formed through both episodes is modeled at $2-5 \times 10^{8} M_{\odot}$, depending on the low-mass part of the IMF (and cannot be more than $\sim 6 \times 10^{8} M_{\odot}$, the total measured stellar mass in the system). The stellar mass formed in the last $10 \mathrm{Myr}$ is thus comparable to or at most double the amount of dense molecular gas remaining, so unless the eventual star formation efficiency is limited to $30-50 \%$, one may ask if the gas can be the raw material for another round of SF.

If the material we trace is indeed forming stars, then the warm molecular medium is likely to impact the stellar IMF, increasing the fraction of high-mass stars by inhibiting the formation of low-mass stars. Theoretical studies of the IMF all involve scaling from a Jeans mass, the mass at which a cloud's self-gravity overcomes its support forces (e.g., Larson 2005). The support can be either simple thermal pressure or largescale turbulent motions. In their recent analytical study, Hennebelle \& Chabrier (2008) note that for typical ISM physical conditions and a reasonable prescription for the turbulence, the turbulent support is more important for the high-mass end of the spectrum, while the evolution of lower-mass condensations are governed by simple thermal support.

The thermal Jeans mass can be written as $M_{\mathrm{J}}=$ $1.1 M_{\odot}(T / 10 K)^{1.5} \rho_{19}^{-0.5}$ (where $\rho_{19}$ is the mass density in units of $10^{-19} \mathrm{~g} \mathrm{~cm}^{-3}$ ) and yields $50 M_{\odot}$ for our median derived temperature $(120 \mathrm{~K})$ and density $\left(10^{4.2} \mathrm{~cm}^{-3}\right)$. This may be indicating that the bulk of the material is indeed unlikely to participate meaningfully in any further SF in its present condition. Of course, the SF will occur in the densest and coolest regions, but they would likely be in approximate pressure equilibrium with the bulk of the gas. If we consider the lowest temperatures allowed by our likelihood analysis, $T \sim 30 \mathrm{~K}$, together with the highest pressures, $\log P \sim 6.7$, then the density is $n=10^{5.2} \mathrm{~cm}^{-3}$, and the Jeans mass is $M_{\mathrm{J}} \sim 3 M_{\odot} 6$

A meaningful comparison with the Galaxy is hampered by the fact that our large-beam M82 observations are necessarily averaging over multiple SF regions, and will include gas in outflows as well as collapsing protostars themselves. Our approach is to examine the material around the Galaxy's most massive SF sites, since they are likely the best Galactic examples of SF on large scales. We consider the sample studied by Leurini et al. (2007) in the millimeter and centimeter-band methanol transitions which are used to derive accurate temperatures and densities. We consider only the envelopes rather than the cores since the cores appear to be heated internally and are presumably already undergoing collapse, and in any case, the envelopes dominate the mass of these regions.

Leurini et al. (2007) find temperatures ranging from $11-36 \mathrm{~K}$, and densities of $10^{5}-10^{6} \mathrm{~cm}^{-3}$. Thus even these massive star formation sites are cooler on average than the dense gas in M82. The lower temperature is not surprising; again, in dense regions the gas temperature will approach the dust temperature, which in the Galaxy ranges from 10-20 K (Paradis et al. 2009). While the inferred thermal pressures in these star forming envelopes are comparable to those we find in M82, the lower temperature and higher density corresponds to a smaller typical Jeans mass - values range from $0.3-2.8 M_{\odot}$, less than the minimum $\sim 3 M_{\odot}$ derived above for M82.

More generally, the characteristic formed stellar mass scale $M_{*}$ is seen to scale as $T_{\min }^{\gamma}$, where $T_{\min }$ is the minimum temperature to which the gas can cool, and the exponent $\gamma$ ranges from 1.7 (obtained in numerical experiments, Jappsen et al. 2005) to 3.35 (via an analytic treatment, Larson 1985). If we take the measured mini-

\footnotetext{
6 We note for completeness that there is evidence for a (massindependent) efficiency factor that relates the mass of a Jeansunstable core to the mass of the actual star which forms from it, believed to be 1.4-2 (Hennebelle \& Chabrier 2008), meaning that the resulting stellar masses are somewhat smaller than the Jeans mass estimates.
} 
mum of $\sim 30 \mathrm{~K}$ versus a conservative $20 \mathrm{~K}$ in the warm Galactic regions, this scaling suggests a factor of at least 2-4 in $M_{*}$ for M82 relative to the Galaxy. Clearly, accurate estimates require theoretical study and more detailed knowledge of the local conditions at the star formation sites, but if the gas we are tracing is indeed involved in star formation, then it likely produces a stellar IMF which is biased against low-mass stars relative to even the massive star formation sites in the Galaxy.

Such a scenario is of course consistent with the reports of low-mass-deficient stellar populations in M82 over the years (Rieke et al. 1980, 1993; Förster Schreiber et al. 2003). Moreover, an IMF biased against low-mass stars produces more luminosity per unit stellar mass than if the IMF is as observed in the Galaxy. Such a topheavy or bottom-light IMF has been proposed to explain an apparent discrepancy between the observed stellar mass buildup and the energy release history in the first half of the Universe $(z>1)$ (Pérez-González et al. 2008; Davé 2008). Given that the typical star forming galaxy in this epoch is now believed to be similar to the local LIRGs and ULIRGs (Le Floc'h et al. 2005; Papovich et al. 2007), the conditions in M82 are likely more indicative of the historical average than those of the Galaxy.

\section{CONCLUSIONS}

We present a study of the dense molecular gas in the starburst nucleus of M82 based on 190-307 GHz spectra toward three positions obtained with the Z-Spec instrument. Z-Spec offers good sensitivity, accurate continuum measurement, and a uniform calibration for spectral lines across this band. We report fluxes for some 20 molecular transitions, many new detections. The measurements of the $J=3 \rightarrow 2$ transitions of $\mathrm{HCO}^{+}, \mathrm{HCN}$, and $\mathrm{HNC}$, and the $J=4 \rightarrow 3$ and $J=5 \rightarrow 4$ transitions of CS motivate an excitation and radiative transfer analysis in which all four species are simultaneously considered, incorporating all of their available published transitions. Our analysis constrains the physical conditions in the dense gas as well as the relative abundances among these species. We trace some $1.7-2.7 \times 10^{8} M_{\odot}$ of gas with $n_{\mathrm{H}_{2}} \simeq 1-3 \times 10^{4} \mathrm{~cm}^{-3}$, and find that it is warm: likely above $50 \mathrm{~K}$ and potentially as high as $500 \mathrm{~K}$, a range which exceeds the level temperature of the transitions studied. The mass and temperature are thus comparable to that found for the warm component in the mid- $J$ CO studies, but the higher density implies a thermal pressure of $1.5-4 \times 10^{6} \mathrm{~K} \mathrm{~cm}^{-3}$, about an order of magnitude higher then inferred from the mid- $J \mathrm{CO}$ transitions.

In the framework of physical and chemical models, the line ratios among $\mathrm{HCN}, \mathrm{HCO}^{+}$, and $\mathrm{HNC}$ indicate that the molecular gas is subject to both UV photons as well as a bulk heating mechanism other than X-rays. A similar conclusion is reached in considering the direct observed cooling in the CO lines up to $J=7 \rightarrow 6$. Cosmic ray heating and dissipation of mechanical energy from the new star clusters are both potential heating sources for the molecular ISM in M82. This feedback has rendered much of the molecular ISM in the nucleus sterile to further SF. We briefly compare the dense molecular gas in M82 with star-forming sites in the Galaxy, concluding that if any of the material we are studying is involved in further SF, then the increased heating likely biases the stellar IMF against low-mass stars, relative to the Galaxy. Such a scenario may be more indicative of the typical SF environment in the Universe's history than the Galactic stellar IMF.

We are deeply grateful to the staff of the Caltech Submillimeter Observatory for their help in Z-Spec's commissioning and observing. We acknowledge Peter Ade and his group for some of our filters and Lionel Duband for the ${ }^{3} \mathrm{He} /{ }^{4} \mathrm{He}$ refrigerator in Z-Spec and are thankful for their help in the early integration of the instrument. We also appreciate the comments and careful reading of an anonymous referee. Finally, we acknowledge the following grants and fellowships: NSF CSO grant (AST0838261) for B. Naylor, NASA SARA grants NAGS11911 and NAGS-12788, an NSF Career grant (AST0239270) and a Research Corporation Award (RI0928) to J. Glenn, a Caltech Millikan and JPL Director's fellowships to C M. Bradford, an NSF grant (AST-0807990) and an NRAO Jansky fellowship to J. Aguirre, and NASA GSRP fellowships to L. Earle and J. Kamenetzky.

The research described in this paper was carried out at the Jet Propulsion Laboratory, California Institute of Technology, under a contract with the National Aeronautics and Space Administration. (c) 2010. All rights reserved.

\section{REFERENCES}

Bayet, E., Aladro, R., Martín, S., Viti, S., \& Martín-Pintado, J. 2009, ApJ, 707, 126

Bayet, E., Lintott, C., Viti, S., Martín-Pintado, J., Martín, S.,

Williams, D. A., \& Rawlings, J. M. C. 2008, ApJ, 685, L35

Boselli, A., Lequeux, J., \& Gavazzi, G. 2002, A\&A, 384, 33

Bradford, C. M., Nikola, T., Stacey, G. J., Bolatto, A. D.,

Jackson, J. M., Savage, M. L., Davidson, J. A., \& Higdon, S. J. 2003, ApJ, 586, 891

Bradford, C. M., Stacey, G. J., Nikola, T., Bolatto, A. D. Jackson, J. M., Savage, M. L., \& Davidson, J. A. 2005, ApJ, 623,866

Bradford, C. M., et al. 2004, in Society of Photo-Optical Instrumentation Engineers (SPIE) Conference Series, Vol. 5498, Society of Photo-Optical Instrumentation Engineers (SPIE) Conference Series, ed. C. M. Bradford, P. A. R. Ade, J. E.

Aguirre, J. J. Bock, M. Dragovan, L. Duband, L. Earle

J. Glenn, H. Matsuhara, B. J. Naylor, H. T. Nguyen, M. Yun, \& J. Zmuidzinas, 257-+

Bradford, C. M., et al. 2009, ApJ, 705, 112

Brand, J., Cesaroni, R., Palla, F., \& Molinari, S. 2001, Astronomy and Astrophysics, 370, 230

Burton, M. G., Hollenbach, D. J., \& Tielens, A. G. G. M. 1990, ApJ, 365, 620

Carlstrom, J. E., \& Kronberg, P. P. 1991, Astrophysical Journal, 366,422

Casasola, V., Combes, F., Bettoni, D., \& Galletta, G. 2007, A\&A, 473,771

Christopher, M. H., Scoville, N. Z., Stolovy, S. R., \& Yun, M. S. 2005, Astrophysical Journal, 622, 346

Colbert, J. W., et al. 1999, Astrophysical Journal, 511, 721

Dame, T. M., Hartmann, D., \& Thaddeus, P. 2001, ApJ, 547, 792

Davé, R. 2008, MNRAS, 385, 147

Earle, L. 2008, PhD thesis, University of Colorado at Boulder

Earle, L., et al. 2006, in Society of Photo-Optical Instrumentation Engineers (SPIE) Conference Series, Vol. 6275, Society of Photo-Optical Instrumentation Engineers (SPIE) Conference Series

Falgarone, E., \& Puget, J. 1995, A\&A, 293, 840

Förster Schreiber, N. M., Genzel, R., Lutz, D., \& Sternberg, A. 2003, ApJ, 599, 193

Fuente, A., García-Burillo, S., Gerin, M., Teyssier, D., Usero, A., Rizzo, J. R., \& de Vicente, P. 2005, Astrophysical Journal Letters, 619, L155

Fuente, A., et al. 2008, A\&A, 492, 675

Gao, Y., \& Solomon, P. M. 2004a, ApJS, 152, 63 
-. 2004b, ApJ, 606, 271

Glenn, J., et al. 2007, in Astronomical Society of the Pacific Conference Series, Vol. 375, From Z-Machines to ALMA: (Sub)Millimeter Spectroscopy of Galaxies, ed. A. J. Baker, J. Glenn, A. I. Harris, J. G. Mangum, \& M. S. Yun , 63-+

Hailey-Dunsheath, S., Nikola, T., Stacey, G. J., Oberst, T. E. Parshley, S. C., Bradford, C. M., Ade, P. A. R., \& Tucker, C. E. 2008, ApJ, 689, L109

Harris, A. I., Stutzki, J., Genzel, R., Lugten, J. B., Stacey, G. J., \& Jaffe, D. T. 1987, ApJ, 322, L49

Henkel, C., Schilke, P., \& Mauersberger, R. 1988, Astronomy and Astrophysics, 201, L23

Hennebelle, P., \& Chabrier, G. 2008, ApJ, 684, 395

Herrmann, F., Madden, S. C., Nikola, T., Poglitsch, A., Timmermann, R., Geis, N., Townes, C. H., \& Stacey, G. J. 1997, ApJ, 481, 343

Huettemeister, S., Henkel, C., Mauersberger, R., Brouillet, N., Wiklind, T., \& Millar, T. J. 1995, Astronomy and Astrophysics, 295, 571

Hughes, D. H., Gear, W. K., \& Robson, E. I. 1994, Monthly Notices of the Royal Astronomical Society, 270, 641

Hunter, S. D., et al. 1997, ApJ, 481, 205

Jappsen, A., Klessen, R. S., Larson, R. B., Li, Y., \& Mac Low, M. 2005, A\&A, 435, 611

Kaufman, M. J., Wolfire, M. G., Hollenbach, D. J., \& Luhman, M. L. 1999, ApJ, 527, 795

Knudsen, K. K., Walter, F., Weiss, A., Bolatto, A., Riechers, D. A., \& Menten, K. 2007, Astrophysical Journal, 666, 156

Koester, B., Stoerzer, H., Stutzki, J., \& Sternberg, A. 1994, A\&A 284,545

Larson, R. B. 1985, MNRAS, 214, 379

-. 2005, MNRAS, 359, 211

Le Floc'h, E., et al. 2005, ApJ, 632, 169

Leeuw, L. L., \& Robson, E. I. 2009, AJ, 137, 517

Leurini, S., Schilke, P., Wyrowski, F., \& Menten, K. M. 2007, A\&A, 466, 215

Lique, F., \& Spielfiedel, A. 2007, Astronomy and Astrophysics, 462, 1179

Lique, F., Spielfiedel, A., \& Cernicharo, J. 2006, Astronomy and Astrophysics, 451, 1125

Loenen, A. F., Spaans, M., Baan, W. A., \& Meijerink, R. 2008, A\&A, 488, L5

Mac Low, M. 1999, ApJ, 524, 169

Maloney, P., \& Black, J. H. 1988, ApJ, 325, 389

Maloney, P. R., Hollenbach, D. J., \& Tielens, A. G. G. M. 1996 ApJ, 466, 561

Mao, R. Q., Henkel, C., Schulz, A., Zielinsky, M., Mauersberger, R., Störzer, H., Wilson, T. L., \& Gensheimer, P. 2000, Astronomy and Astrophysics, 358, 433

Martín, S., Martín-Pintado, J., \& Mauersberger, R. 2009, ApJ, 694, 610

Mauersberger, R., \& Henkel, C. 1989, Astronomy and Astrophysics, 223, 79

Mauersberger, R., Henkel, C., Walmsley, C. M., Sage, L. J., \& Wiklind, T. 1991, Astronomy and Astrophysics, 247, 307

Meijerink, R., \& Spaans, M. 2005, A\&A, 436, 397

Meijerink, R., Spaans, M., \& Israel, F. P. 2007, A\&A, 461, 793

Meixner, M., \& Tielens, A. G. G. M. 1993, ApJ, 405, 216

Morris, M., Palmer, P., Turner, B. E., \& Zuckerman, B. 1974, ApJ, 191, 349

Mühle, S., Seaquist, E. R., \& Henkel, C. 2007, ApJ, 671, 1579

Nakai, N., \& Kuno, N. 1995, PASJ, 47, 761

Naylor, B. J., et al. 2003, in Society of Photo-Optical

Instrumentation Engineers (SPIE) Conference Series, Vol. 4855, Society of Photo-Optical Instrumentation Engineers (SPIE)

Conference Series, ed. T. G. Phillips \& J. Zmuidzinas, 239-248
Nguyen-Q-Rieu, Nakai, N., \& Jackson, J. M. 1989, Astronomy and Astrophysics, 220, 57

Nikolić, S., Johansson, L. E. B., \& Harju, J. 2003, Astronomy and Astrophysics, 409, 941

Paglione, T. A. D., Jackson, J. M., Ishizuki, S., \& Rieu, N. 1995, AJ, 109,1716

Pan, L., \& Padoan, P. 2009, ApJ, 692, 594

Panuzzo, P., et al. 2010, ArXiv e-prints

Papovich, C., et al. 2007, ApJ, 668, 45

Paradis, D., Bernard, J., \& Mény, C. 2009, A\&A, 506, 745

Pérez-González, P. G., et al. 2008, ApJ, 675, 234

Rieke, G. H., Lebofsky, M. J., Thompson, R. I., Low, F. J., \& Tokunaga, A. T. 1980, ApJ, 238, 24

Rieke, G. H., Loken, K., Rieke, M. J., \& Tamblyn, P. 1993, ApJ 412,99

Sakai, S., \& Madore, B. F. 1999, Astrophysical Journal, 526, 599

Sanders, D. B., Mazzarella, J. M., Kim, D.-C., Surace, J. A., \&

Soifer, B. T. 2003, Astronomical Journal, 126, 1607

Satyapal, S., Watson, D. M., Pipher, J. L., Forrest, W. J., Greenhouse, M. A., Smith, H. A., Fischer, J., \& Woodward, C. E. 1997, ApJ, 483, 148

Schmidt-Burgk, J. 1990, in Astrophysics and Space Science Library, Vol. 158, Submillimetre Astronomy, ed. G. D. Watt \& A. S. Webster, $11-12$

Schöier, F. L., van der Tak, F. F. S., van Dishoeck, E. F., \&

Black, J. H. 2005, Astronomy and Astrophysics, 432, 369

Seaquist, E. R., \& Frayer, D. T. 2000, Astrophysical Journal, 540 765

Seaquist, E. R., Lee, S. W., \& Moriarty-Schieven, G. H. 2006, ApJ, 638, 148

Solomon, P. M., Downes, D., \& Radford, S. J. E. 1992, Astrophysical Journal Letters, 398, L29

Stacey, G. J., Jaffe, D. T., Geis, N., Grenzel, R., Harris, A. I., Poglitsch, A., Stutzki, J., \& Townes, C. H. 1993, ApJ, 404, 219

Strickland, D. K., \& Heckman, T. M. 2007, ApJ, 658, 258

Stutzki, J., Stacey, G. J., Genzel, R., Harris, A. I., Jaffe, D. T., \& Lugten, J. B. 1988, ApJ, 332, 379

Suchkov, A., Allen, R. J., \& Heckman, T. M. 1993, ApJ, 413, 542

Thuma, G., Neininger, N., Klein, U., \& Wielebinski, R. 2000, Astronomy and Astrophysics, 358, 65

Tielens, A. G. G. M., \& Hollenbach, D. 1985, ApJ, 291, 722

Turner, B. E., Zuckerman, B., Palmer, P., \& Morris, M. 1973, ApJ, 186, 123

van der Tak, F. F. S., Aalto, S., \& Meijerink, R. 2008, A\&A, 477, L5

VERITAS Collaboration et al. 2009, Nature, 462, 770

Ward, J. S. 2002, PhD thesis, California Institute of Technology

Ward, J. S., Zmuidzinas, J., Harris, A. I., \& Isaak, K. G. 2003, Astrophysical Journal, 587, 171

Weiß, A., Neininger, N., Hüttemeister, S., \& Klein, U. 2001, Astronomy and Astrophysics, 365, 571

Weiß, A., Walter, F., \& Scoville, N. Z. 2005, A\&A, 438, 533

Wild, W., Harris, A. I., Eckart, A., Genzel, R., Graf, U. U. Jackson, J. M., Russell, A. P. G., \& Stutzki, J. 1992,

Astronomy and Astrophysics, 265, 447

Wilson, C. D. 1995, ApJ, 448, L97+

Wolfire, M. G., Tielens, A. G. G. M., \& Hollenbach, D. 1990 ApJ, 358, 116

Wu, J., Evans, II, N. J., Gao, Y., Solomon, P. M., Shirley, Y. L., \& Vanden Bout, P. A. 2005, ApJ, 635, L173

Yao, L. 2009, ApJ, 705, 766

Yao, L., Bell, T. A., Viti, S., Yates, J. A., \& Seaquist, E. R. 2006, ApJ, 636, 881

Yun, M. S., Ho, P. T. P., \& Lo, K. Y. 1994, Nature, 372, 530 